\title{
Palladium-Catalyzed Cross-Coupling of Acyl Chlorides and Organostannanes
}

\author{
Rachel Lerebours, Alejandra Camacho-Soto, Christian Wolf* \\ Department of Chemistry, Georgetown University, Washington, DC 20057 \\ cw27@georgetown.edu
}

\section{Table of Contents}

Synthetic procedure

Compound purification and characterization

${ }^{1} \mathrm{H}$ NMR and ${ }^{13}$ NMR spectra of all cross-coupling products

References
Page:

S2

S2

S6

S26 
General coupling procedure. A mixture of acyl chloride (1.4 mmol), PXPd (2.5 mol\%), organostannane $(1.5 \mathrm{mmol})$ in $5 \mathrm{~mL}$ of anhydrous acetonitrile was stirred under nitrogen at $82{ }^{\circ} \mathrm{C}$ for $20 \mathrm{~h}$. The reaction mixture was allowed to cool to room temperature, quenched with water and extracted with methylene chloride. The combined organic layers were washed with brine, dried over $\mathrm{MgSO}_{4}$ and the solvents were removed under vacuum. The residue was purified by flash chromatography on silica gel as discussed below.

Benzophenone (3). ${ }^{1}$ Flash chromatography (5:1 hexanes:ethyl acetate) gave white solids (98\%), mp 48-49.5 ${ }^{\circ} \mathrm{C} .{ }^{1} \mathrm{H}$ NMR: $\delta$ 7.43-7.51 (m, 4H), 7.56-7.59 (m, 2H), 7.80-7.83 (m, 4H). ${ }^{13} \mathrm{C}$ NMR $\delta 128.6,130.3,132.7,137.8,197.1$.

Phenyl phenylacetylene ketone (5). ${ }^{2}$ Flash chromatography (2:1 hexanes:methylene chloride) provided a colorless oil (93\%). ${ }^{1} \mathrm{H}$ NMR: $\delta$ 7.40-7.44 (m, 2H), 7.47-7.54 (m, 3H), 7.63 (m, 1H), 7.68-7.70 (m, 2H), 8.22-8.24 (m, 2H). ${ }^{13} \mathrm{C}$ NMR $\delta 87.3,91.6,120.6,129.1,130.0,131.3,133.5$, $134.5,137.4,178.5$.

2-Furan 3-methylphenyl ketone (8). ${ }^{3}$ Purification by flash chromatography (2:1 hexanes:methylene chloride) provided a colorless oil (92\%). ${ }^{1} \mathrm{H}$ NMR: $\delta 2.46$ (s, 3H), 6.60 (m, 1H), $7.24(\mathrm{~d}, J=3.6 \mathrm{~Hz}, 1 \mathrm{H}), 7.40-7.43(\mathrm{~m}, 2 \mathrm{H}), 7.73-7.79(\mathrm{~m}, 3 \mathrm{H}) .{ }^{13} \mathrm{C}$ NMR $\delta 21.6,112.4$, $120.8,126.7,128.5,129.9,133.6,137.5,138.5,147.3,152.5,183.1$.

4-Cyanophenyl 2-furan ketone (10). Purification by flash chromatography (1:1 diethyl ether:hexanes) gave yellow crystals (96\%), mp 136-138 ${ }^{\circ} \mathrm{C} .{ }^{1} \mathrm{H}$ NMR: $\delta 6.67(\mathrm{dd}, J=1.0 \mathrm{~Hz}, 3.4$ Hz, 1H), 7.33 (d, J=3.4 Hz, 1H), 7.76 (d, $J=1.0 \mathrm{~Hz}, 1 \mathrm{H}) .7 .83$ (d, $J=8.2 \mathrm{~Hz}, 2 \mathrm{H}), 8.10$ (d, $J=8.2$ Hz, 2H). ${ }^{13} \mathrm{C}$ NMR $\delta 113.0,116.1,118.2,121.5,130.0,132.5,140.8,148.1,152.1,180.9$. Anal Calcd for $\mathrm{C}_{12} \mathrm{H}_{7} \mathrm{NO}_{2}$ : C, 73.09; H, 3.58; N, 7.10. Found: C, 73.19; H, 3.56; N, 6.91. 
4-Cyanophenyl phenylacetylene ketone (11). ${ }^{4}$ Purification by flash chromatography $(1: 1$ hexanes:methylene chloride) provided yellow crystals $(98 \%), \mathrm{mp}=139-140{ }^{\circ} \mathrm{C} .{ }^{1} \mathrm{H}$ NMR: $\delta$ 7.45-7.56 (m, 3H), 7.71-7.74 (m, 2H), 7.84-7.87 (m, 2H), 8.33-8.35 (m, 2H). ${ }^{13} \mathrm{C}$ NMR $\delta 95.2$, 107.2, 117.6, 118.2, 119.9, 129.1, 130.1, 131.7, 132.8, 133.5, 140.0, 176.5.

2-Furan 4-methoxyphenyl ketone (13). ${ }^{5}$ Purification by flash chromatography (2:1 hexanes:methylene chloride) provided a colorless oil $(67 \%) .{ }^{1} \mathrm{H}$ NMR: $\delta 3.88(\mathrm{~s}, 3 \mathrm{H}), 6.57(\mathrm{~m}$, 1H), $6.98(\mathrm{~m}, 2 \mathrm{H}), 7.22(\mathrm{~d}, J=3.4 \mathrm{~Hz}, 1 \mathrm{H}), 7.68(\mathrm{~m}, 1 \mathrm{H}), 8.03(\mathrm{~m}, 2 \mathrm{H}) .{ }^{13} \mathrm{C}$ NMR $\delta$ 55.7, 112.3, $113.9,119.8,130.1,131.9,146.7,153.5,163.5,181.9$.

2-Furan piperonyloyl ketone (15). Purification by flash chromatography (2:1 hexanes:ethyl acetate) gave a yellow oil ( $82 \%) .{ }^{1} \mathrm{H}$ NMR: $\delta 6.06(\mathrm{~s}, 2 \mathrm{H}), 6.58(\mathrm{~m}, 1 \mathrm{H}), 6.91(\mathrm{~d}, J=8.2 \mathrm{~Hz}, 1 \mathrm{H})$, $7.24(\mathrm{~m}, 1 \mathrm{H}), 7.52(\mathrm{~m}, 1 \mathrm{H}), 7.67-7.69(\mathrm{~m}, 2 \mathrm{H}) .{ }^{13} \mathrm{C}$ NMR $\delta 102.1,108.2,109.6,112.3,120.1$, 125.9, 131.9, 146.9, 148.3, 151.8, 152.4, 181.3. Anal Calcd for $\mathrm{C}_{12} \mathrm{H}_{8} \mathrm{O}_{4}: \mathrm{C}, 66.67 ; \mathrm{H}, 3.73$. Found: C, 66.52; H, 3.77.

Piperonyloyl 2-thiophen ketone (17). Purification by flash chromatography (1:1 hexanes:methylylene chloride) gave a yellow oil (81 \%). ${ }^{1} \mathrm{H}$ NMR: $\delta 6.08(\mathrm{~s}, 2 \mathrm{H}), 6.90(\mathrm{~d}, J=7.8$ $\mathrm{Hz}, 1 \mathrm{H}), 7.16$ (dd, $J=3.5 \mathrm{~Hz}, 4.8 \mathrm{~Hz}, 1 \mathrm{H}), 7.39$ (d, $J=1.7 \mathrm{~Hz}, 1 \mathrm{H}), 7.50(\mathrm{dd}, J=1.7 \mathrm{~Hz}, 7.8 \mathrm{~Hz}$, 1H), $7.65(\mathrm{~d}, J=3.5 \mathrm{~Hz}, 1 \mathrm{H}), 7.68(\mathrm{~d}, J=4.8 \mathrm{~Hz}, 1 \mathrm{H}) .{ }^{13} \mathrm{C}$ NMR $\delta 102.1,108.1,109.7,125.7$, 128.1, 132.6, 133.9, 134.4, 143.7, 148.2, 151.4, 186.3. Anal Calcd for $\mathrm{C}_{12} \mathrm{H}_{8} \mathrm{O}_{3} \mathrm{~S}: \mathrm{C}, 62.06 ; \mathrm{H}$, 3.47. Found: C 61.94; H, 3.47.

Phenylacetylene piperonyloyl ketone (18). ${ }^{6}$ Purification by flash chromatography (1:1 hexanes:methylylene chloride) gave a colorless oil $(76 \%) .{ }^{1} \mathrm{H}$ NMR: $\delta 6.06(\mathrm{~s}, 2 \mathrm{H}), 6.89(\mathrm{~d}$, $J=8.1 \mathrm{~Hz}, 1 \mathrm{H}), 7.39-7.68(\mathrm{~m}, 5 \mathrm{H}), 7.88(\mathrm{~m}, 2 \mathrm{H}) .{ }^{13} \mathrm{C}$ NMR $\delta 86.7,92.2,102.0,107.9,108.2$, $120.2,127.1,128.6,130.7,132.0,132.8,148.2,152.8,176.0$. 
Propyl 2-thiophen ketone (20). ${ }^{7}$ Flash chromatography (1:1 hexanes:methylene chloride) provided a yellow oil (78 \%). ${ }^{1} \mathrm{H}$ NMR: 1.02 (t, $\left.J=7.5 \mathrm{~Hz}, 3 \mathrm{H}\right), 1.81$ (m, 2H), 2.89 (t, J=7.5 Hz, 2H), $7.13(\mathrm{dd}, J=3.5 \mathrm{~Hz}, 4.0 \mathrm{~Hz}, 1 \mathrm{H}), 7.62(\mathrm{~d}, J=1.2 \mathrm{~Hz}, 4.0 \mathrm{~Hz}, 1 \mathrm{H}), 7.72(\mathrm{dd}, J=1.2 \mathrm{~Hz}, 3.5 \mathrm{~Hz}$, 1H). ${ }^{13} \mathrm{C}$ NMR $\delta 14.1,18.5,41.5,128.3,131.9,133.6,144.8,193.6$.

2-Pivaloylfuran (22). ${ }^{8}$ Purification by flash chromatography (2:1 hexanes:methylene chloride) provided a colorless oil (85\%). ${ }^{1} \mathrm{H}$ NMR: $\delta 1.36(\mathrm{~s}, 9 \mathrm{H}), 6.51(\mathrm{~m}, 1 \mathrm{H}), 7.21(\mathrm{~d}, J=4.6 \mathrm{~Hz}, 1 \mathrm{H})$, $7.55(\mathrm{~m}, 1 \mathrm{H}) .{ }^{13} \mathrm{C}$ NMR $\delta 28.1,43.2,111.9,118.2,145.2,152.7,194.9$.

4-Chlorophenyl 2-furan ketone (24). ${ }^{9}$ Purification by flash chromatography (3:1 hexanes:ethyl acetate) gave a white solid material $(80 \%)$, mp 98-99 ${ }^{\circ} \mathrm{C} .{ }^{1} \mathrm{H}$ NMR: $\delta 6.61(\mathrm{~d}, J=3.6 \mathrm{~Hz}, 1 \mathrm{H})$, $7.27(\mathrm{dd}, J=0.6 \mathrm{~Hz}, 3.6 \mathrm{~Hz}, 1 \mathrm{H}), 7.48(\mathrm{~d}, J=8.7 \mathrm{~Hz}, 2 \mathrm{H}), 7.71$ (d, $J=0.6 \mathrm{~Hz}, 1 \mathrm{H}), 7.95$ (d, $J=8.7$ $\mathrm{Hz}, 2 \mathrm{H}) .{ }^{13} \mathrm{C}$ NMR $\delta 112.7,120.9,129.0,131.0,135.9,139.4,147.5,152.3,181.4$.

3-Chlorophenyl 2-furan ketone (28). ${ }^{9}$ Purification by flash chromatography (3:1 hexanes:ethyl acetate) gave a yellow oil (80 \%). ${ }^{1} \mathrm{H}$ NMR: $\delta 6.64(\mathrm{~m}, 1 \mathrm{H}), 7.29(\mathrm{~d}, J=3.6 \mathrm{~Hz}, 1 \mathrm{H}), 7.46(\mathrm{dd}$, $J=7.6 \mathrm{~Hz}, 9.2 \mathrm{~Hz}, 1 \mathrm{H}), 7.57(\mathrm{~m}, 1 \mathrm{H}), 7.75(\mathrm{~m}, 1 \mathrm{H}), 7.9$ (d, $J=7.6 \mathrm{~Hz}, 1 \mathrm{H}), 7.99(\mathrm{~m}, 1 \mathrm{H}) .{ }^{13} \mathrm{C}$ NMR $\delta 112.7,121.2,127.7,129.6,130.0,132.8,135.1,139.2,147.7,152.1,181.4$.

2-Chlorophenyl 2-furan ketone (30). ${ }^{9}$ Purification by flash chromatography $(2: 1$ hexanes:methylene chloride) gave a brown oil (87\%). ${ }^{1} \mathrm{H}$ NMR: $\delta 6.59(\mathrm{~d}, J=3.4 \mathrm{~Hz}, 1 \mathrm{H}), 7.06$ $(\mathrm{d}, J=3.4 \mathrm{~Hz}, 1 \mathrm{H}), 7.37-7.48(\mathrm{~m}, 4 \mathrm{H}), 7.71(\mathrm{~s}, 1 \mathrm{H}) .{ }^{13} \mathrm{C}$ NMR $\delta$ 112.9, 121.8, 126.8, 129.4, 130.5, $131.8,138.9,148.3,152.3,182.7$.

4-Chlorophenyl phenylacetylene ketone (31). ${ }^{10}$ Purification by flash chromatography (1:1 hexanes:methylene chloride) provided a yellow oil (67\%). ${ }^{1} \mathrm{H}$ NMR: $\delta$ 7.42-7.53 (m, 5H), 7.70 (d, $J=8.4 \mathrm{~Hz}, 2 \mathrm{H}), 8.17$ (d, $J=8.4 \mathrm{~Hz}, 2 \mathrm{H}) .{ }^{13} \mathrm{C}$ NMR $\delta 87.3,94.1,120.1,129.0,129.3,131.1$, 131.3, 133.4, 135.6, 141.0, 177.2. 
4-Chlorophenyl 2-thiophen ketone (32). ${ }^{9}$ Flash chromatography (2:1 hexanes:ethyl acetate) afforded brown solids (72 \%), mp 75-76 ${ }^{\circ} \mathrm{C} .{ }^{1} \mathrm{H}$ NMR: $\delta 7.19$ (dd, $J=3.7 \mathrm{~Hz}, 4.8 \mathrm{~Hz}, 1 \mathrm{H}$ ), 7.50 $(\mathrm{d}, J=8.5 \mathrm{~Hz}, 2 \mathrm{H}), 7.64(\mathrm{~d}, J=3.7 \mathrm{~Hz}, 1 \mathrm{H}), 7.76(\mathrm{~d}, J=4.8 \mathrm{~Hz}, 1 \mathrm{H}), 7.84(\mathrm{~d}, J=8.5 \mathrm{~Hz}, 2 \mathrm{H}) .{ }^{13} \mathrm{C}$ NMR $\delta 127.5,128.4,129.4,130.1,132.5,134.9,135.1,135.4,139.9,143.3,186.9$.

3-Chlorophenyl 2-thiophen ketone (33). ${ }^{9}$ Flash chromatography (5:1hexanes:methylene chloride) yielded a yellow oil (72\%). ${ }^{1} \mathrm{H}$ NMR: $\delta 7.17(\mathrm{dd}, J=3.9 \mathrm{~Hz}, 4.9 \mathrm{~Hz}, 1 \mathrm{H}), 7.42(\mathrm{dd}$, $J=7.8 \mathrm{~Hz}, 8.3 \mathrm{~Hz}, 1 \mathrm{H}), 7.55(\mathrm{~m}, 1 \mathrm{H}), 7.63(\mathrm{dd}, J=1.2 \mathrm{~Hz}, 3.9 \mathrm{~Hz}, 1 \mathrm{H}), 7.70-7.75(\mathrm{~m}, 2 \mathrm{H}), 7.81$ (m, 1H). ${ }^{13} \mathrm{C}$ NMR $\delta 127.5,128.5,129.3,130.0,132.5,134.9,135.1,135.4,139.9,143.2,186.9$. 4-Bromophenyl 2-furan ketone (35). ${ }^{9}$ Purification by flash chromatography (2:1 hexanes:methylene chloride) provided a yellow oil ( $85 \%$ ). ${ }^{1} \mathrm{H}$ NMR: $\delta 6.60$ (dd, $J=1.8 \mathrm{~Hz}, 3.5$ $\mathrm{Hz}, 1 \mathrm{H}), 7.25(\mathrm{~d}, J=3.5 \mathrm{~Hz}, 1 \mathrm{H}), 7.63(\mathrm{~d}, J=8.8 \mathrm{~Hz}, 2 \mathrm{H}), 7.71(\mathrm{~m}, 1 \mathrm{H}), 7.86(\mathrm{~d}, J=8.8 \mathrm{~Hz}, 2 \mathrm{H})$. ${ }^{13} \mathrm{C}$ NMR $\delta 112.4,120.9,127.9,131.1,131.9,136.3,147.5,152.4,181.4$.

3-Bromophenyl 2-furan ketone (37). ${ }^{11}$ Purification by flash chromatography (1:1 hexanes:methylene chloride) provided a yellow oil (62 \%). ${ }^{1} \mathrm{H}$ NMR: $\delta 6.53(\mathrm{dd}, J=1.9 \mathrm{~Hz}, 3.6$ $\mathrm{Hz}, 1 \mathrm{H}), 7.15(\mathrm{dd}, J=0.8 \mathrm{~Hz}, 3.6 \mathrm{~Hz}, 1 \mathrm{H}), 7.25(\mathrm{~m}, 1 \mathrm{H}), 7.57-7.61(\mathrm{~m}, 2 \mathrm{H}), 7.79(\mathrm{~d}, J=7.8,1 \mathrm{H})$, $7.99(\mathrm{~d}, J=2.1 \mathrm{~Hz}, 1 \mathrm{H}) .{ }^{13} \mathrm{C}$ NMR $\delta 112.4,120.9,122.6,127.8,130.0,132.2,135.4,138.8,147.4$, $151.9,180.7$.

2-Bromophenyl 2-furan ketone (39). ${ }^{9}$ Purification by flash chromatography (1:1 hexanes:methylene chloride) provided a brown oil $(57 \%) .{ }^{1} \mathrm{H}$ NMR: $\delta 6.57(\mathrm{dd}, J=1.7 \mathrm{~Hz}, 3.7$ $\mathrm{Hz}, 1 \mathrm{H}), 7.05(\mathrm{dd}, J=0.7 \mathrm{~Hz}, 3.7 \mathrm{~Hz}, 1 \mathrm{H}), 7.32-7.45(\mathrm{~m}, 3 \mathrm{H}), 7.63-7.70(\mathrm{~m}, 2 \mathrm{H}) .{ }^{13} \mathrm{C} \mathrm{NMR}$ $\delta 112.7,119.9,121.6,127.4,129.4,131.8,133.7,139.7,148.4,152.1,182.8$. 
${ }^{1}$ H NMR and ${ }^{13}$ NMR spectra of all cross-coupling products

NMR spectra were obtained on a Varian FT-NMR spectrometer at $300 \mathrm{MHz}\left({ }^{1} \mathrm{H}\right.$ NMR) and 75 $\mathrm{MHz}\left({ }^{13} \mathrm{C} \mathrm{NMR}\right)$ using $\mathrm{CDCl}_{3}$ as the solvent. Chemical shifts are reported in ppm relative to TMS.

${ }^{1} \mathrm{H}$ NMR and ${ }^{13}$ NMR spectra of Benzophenone (3).

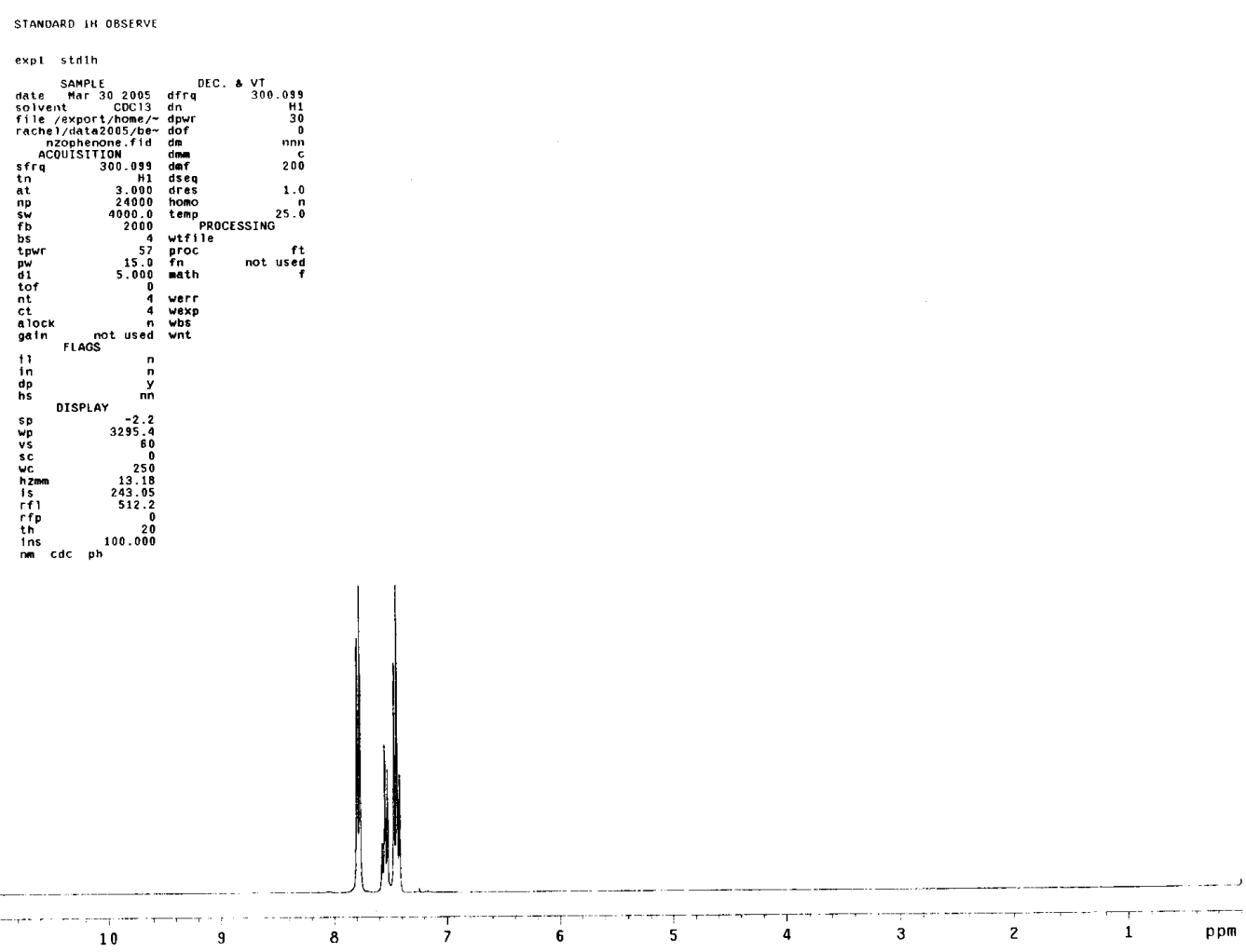




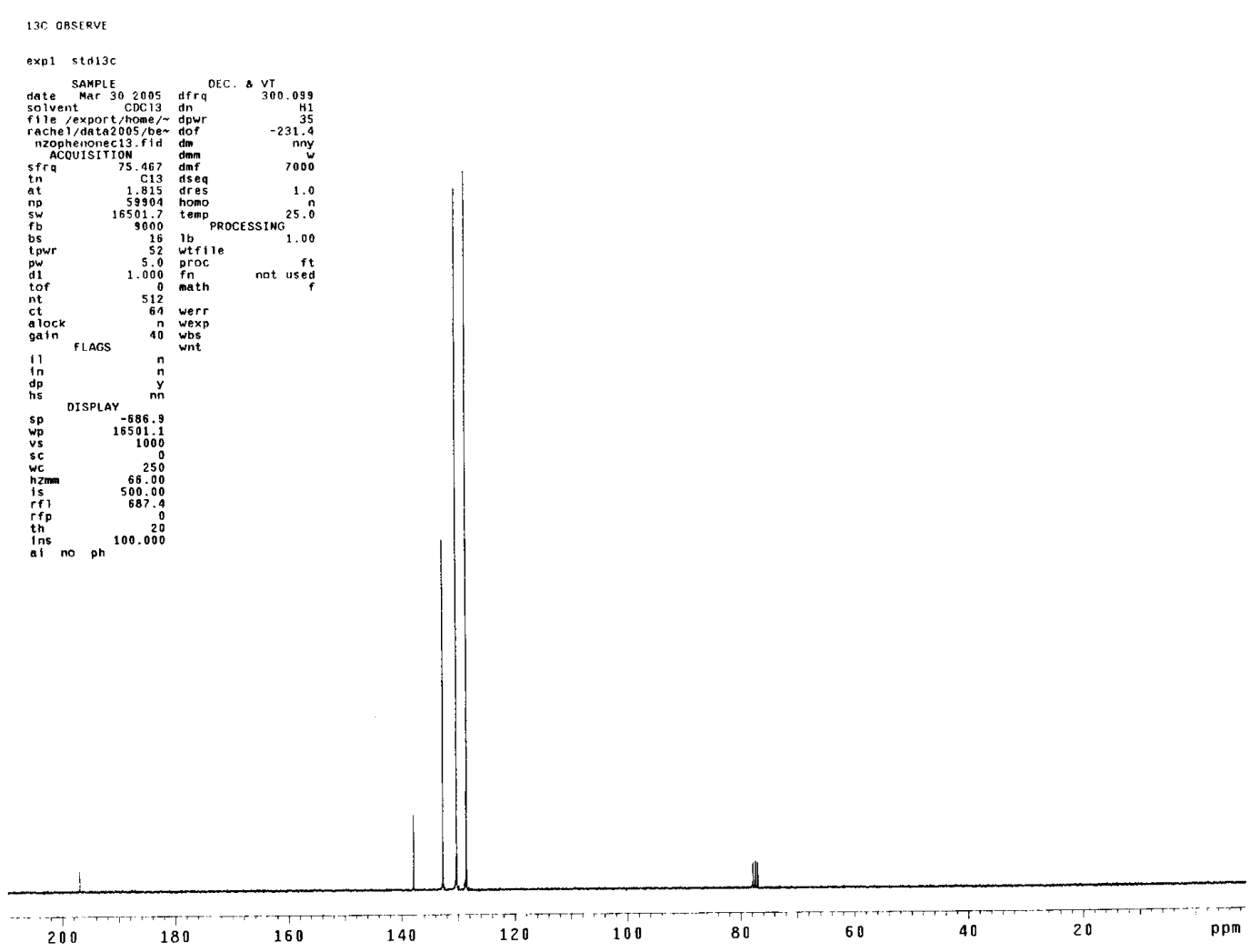

${ }^{1}$ H NMR and ${ }^{13}$ NMR spectra of Phenyl phenylacetylene ketone (5).

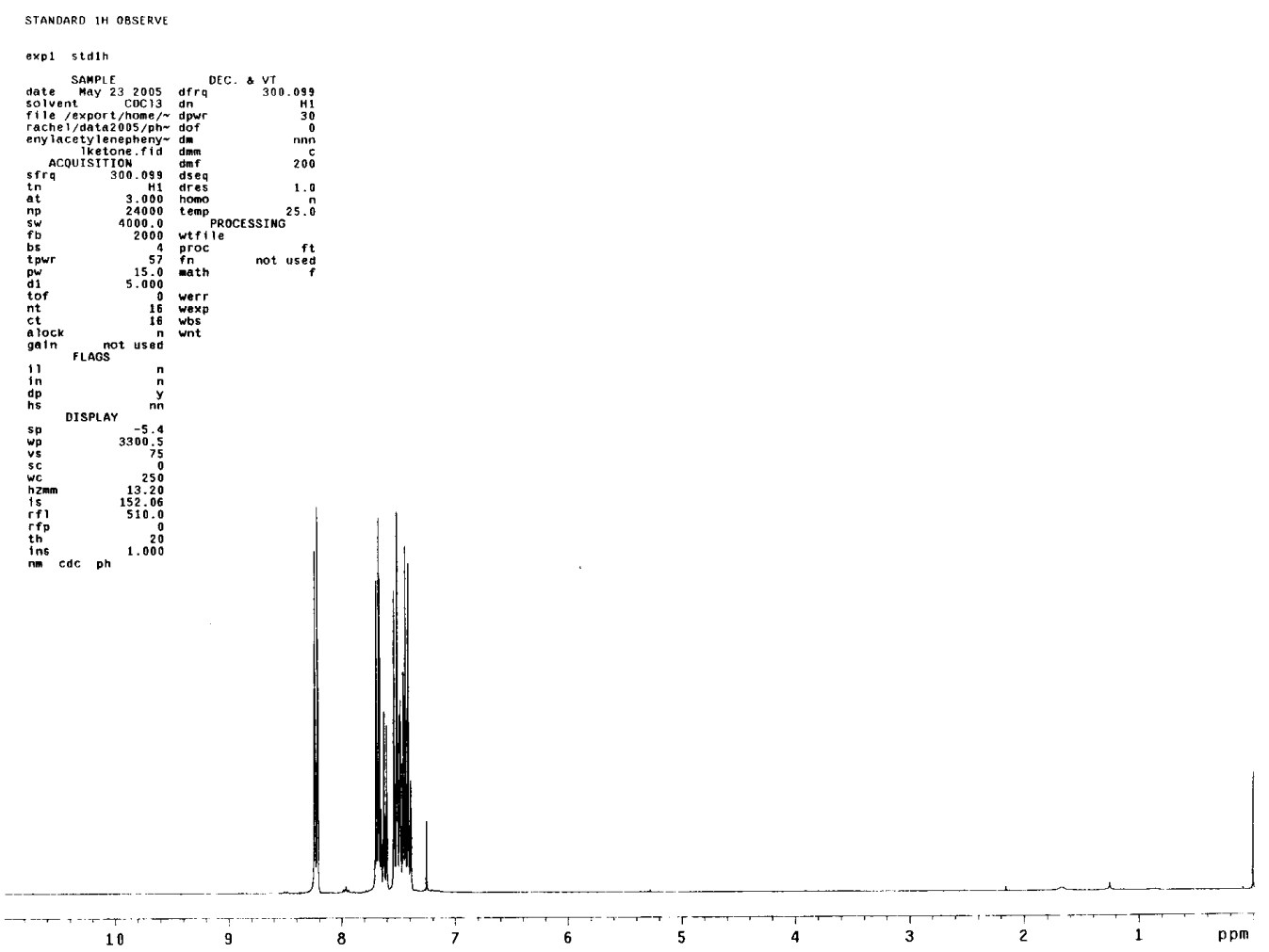




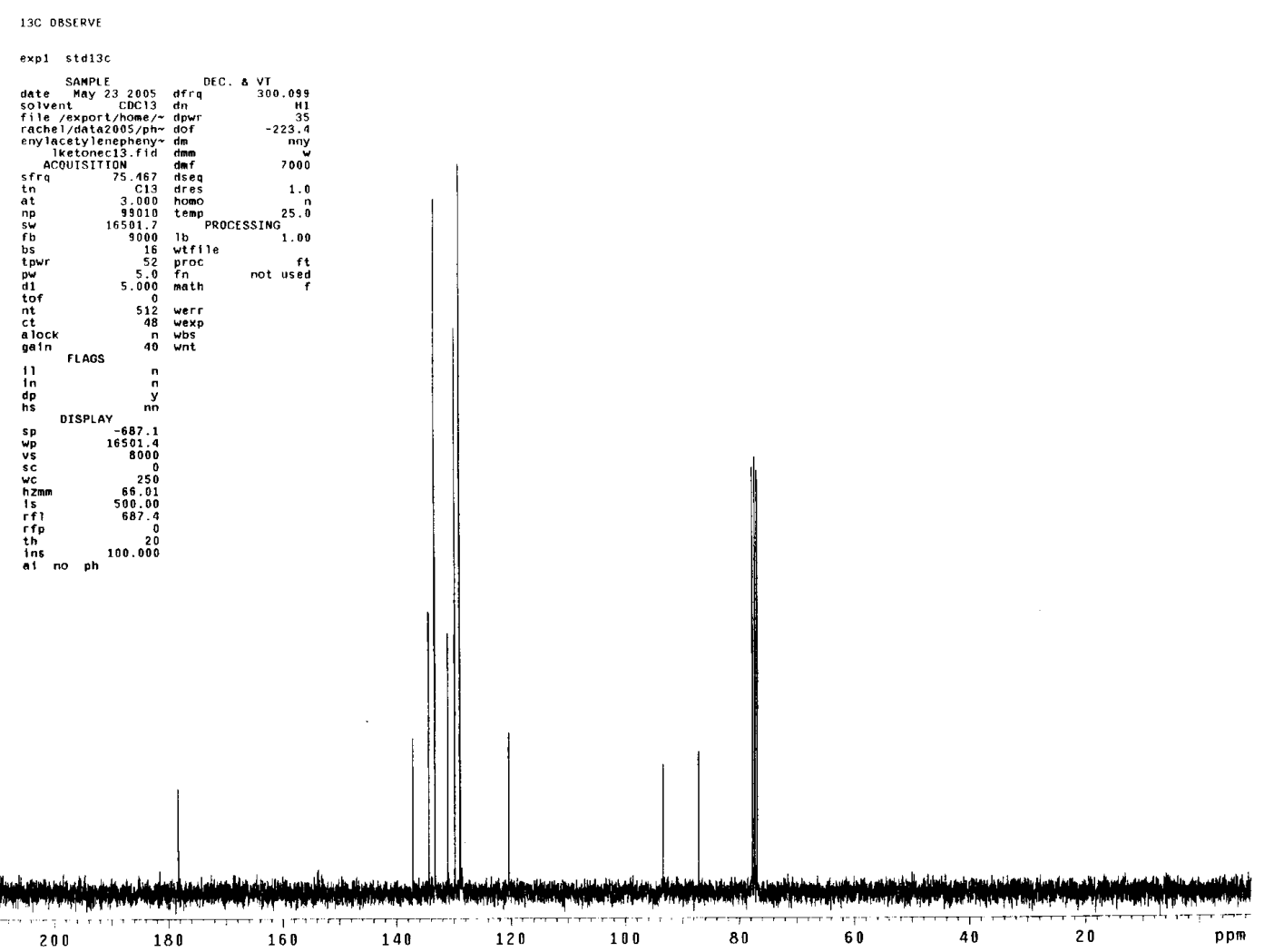

${ }^{1}$ H NMR and ${ }^{13}$ NMR spectra of 2-Furan 3-methylphenyl ketone (8).
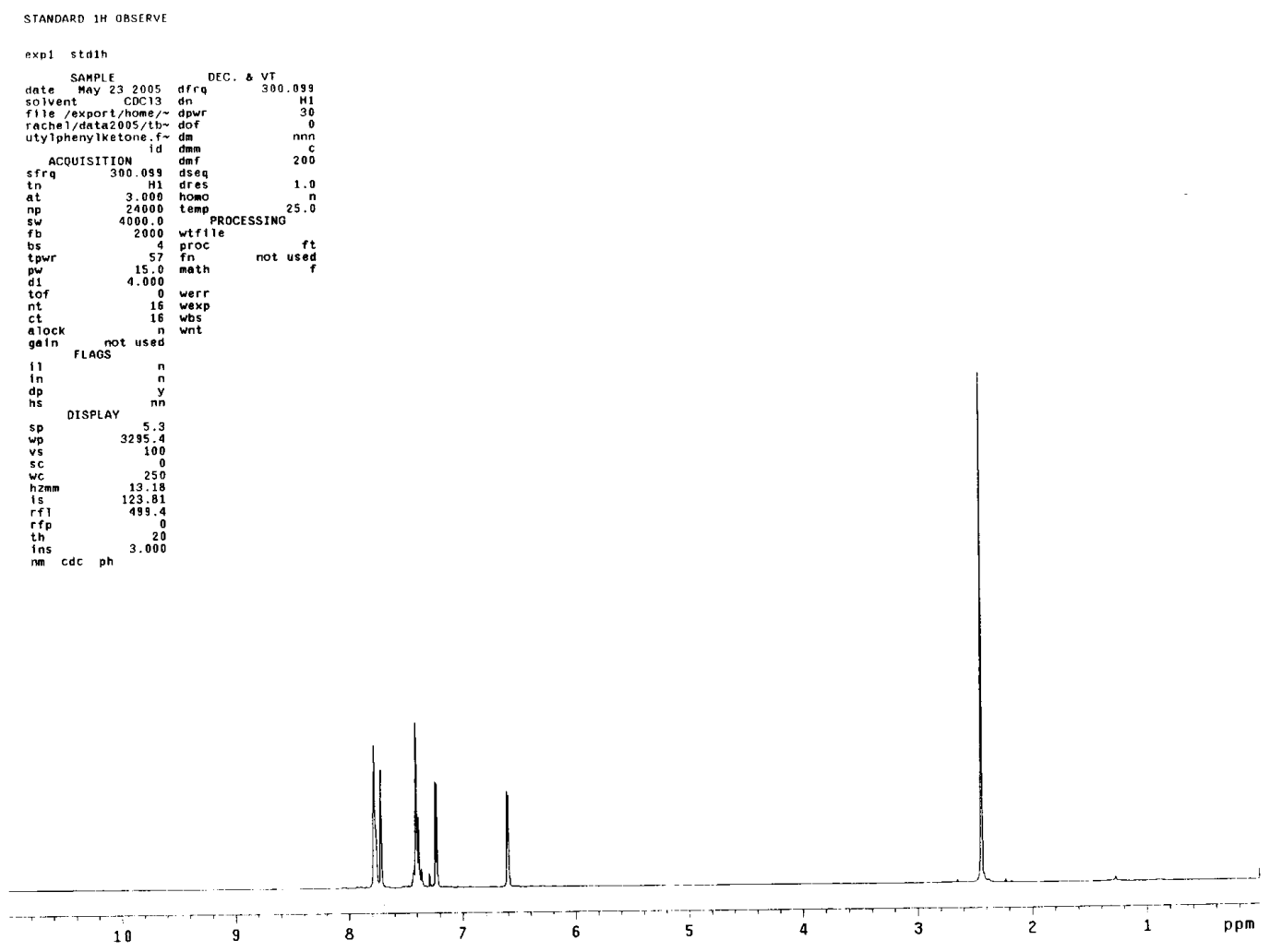


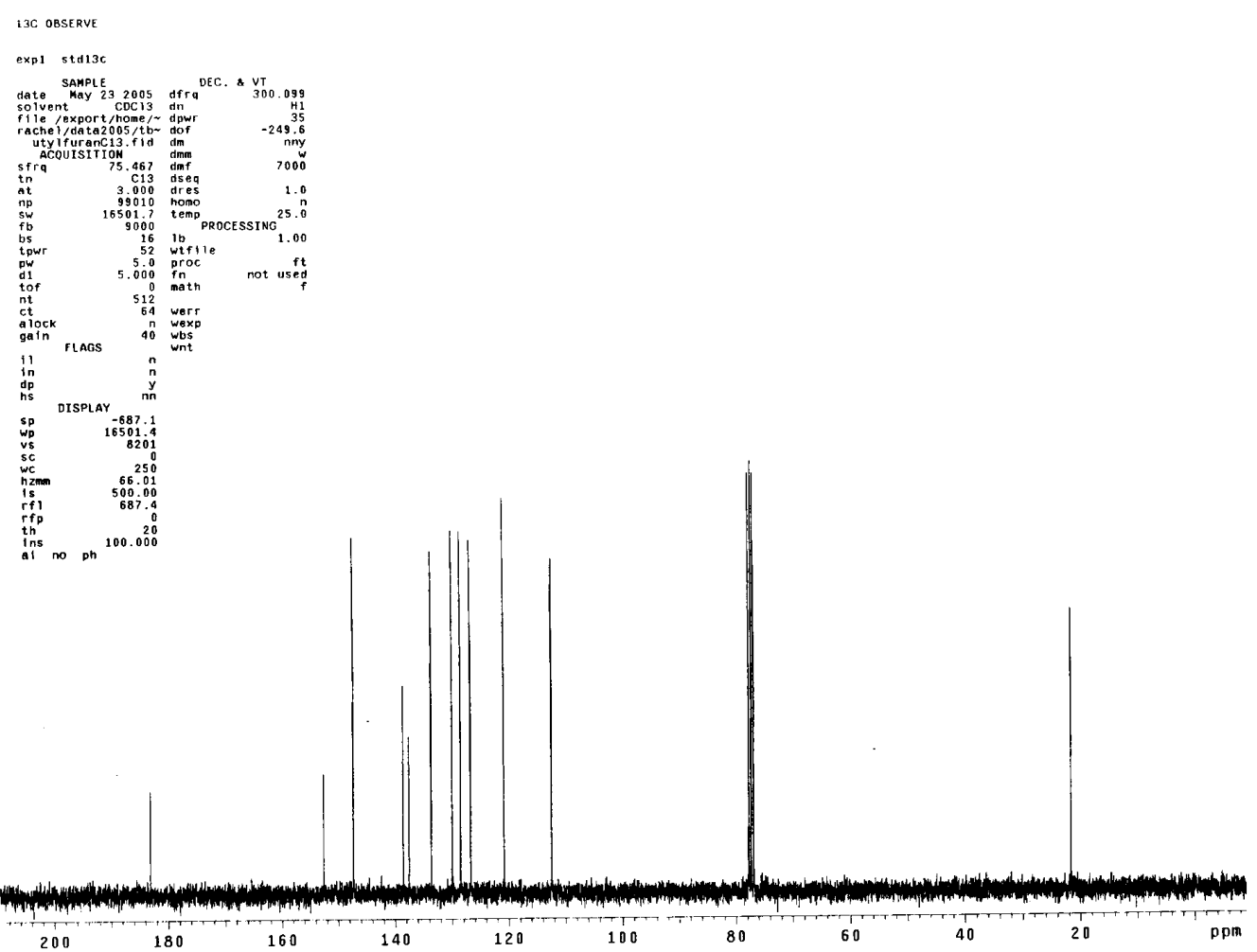

${ }^{1}$ H NMR and ${ }^{13}$ NMR spectra of 4-Cyanophenyl 2-furan ketone (10).
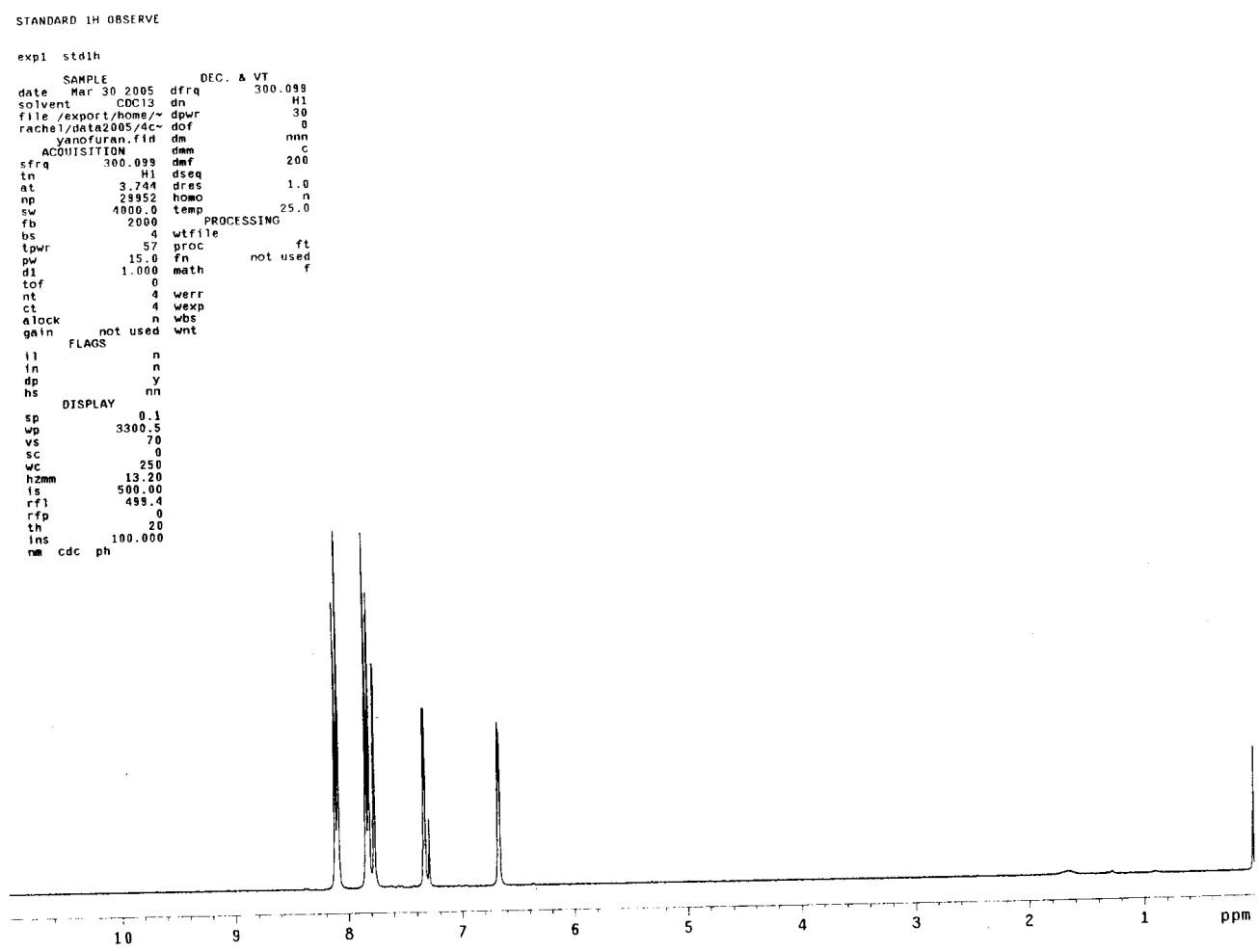


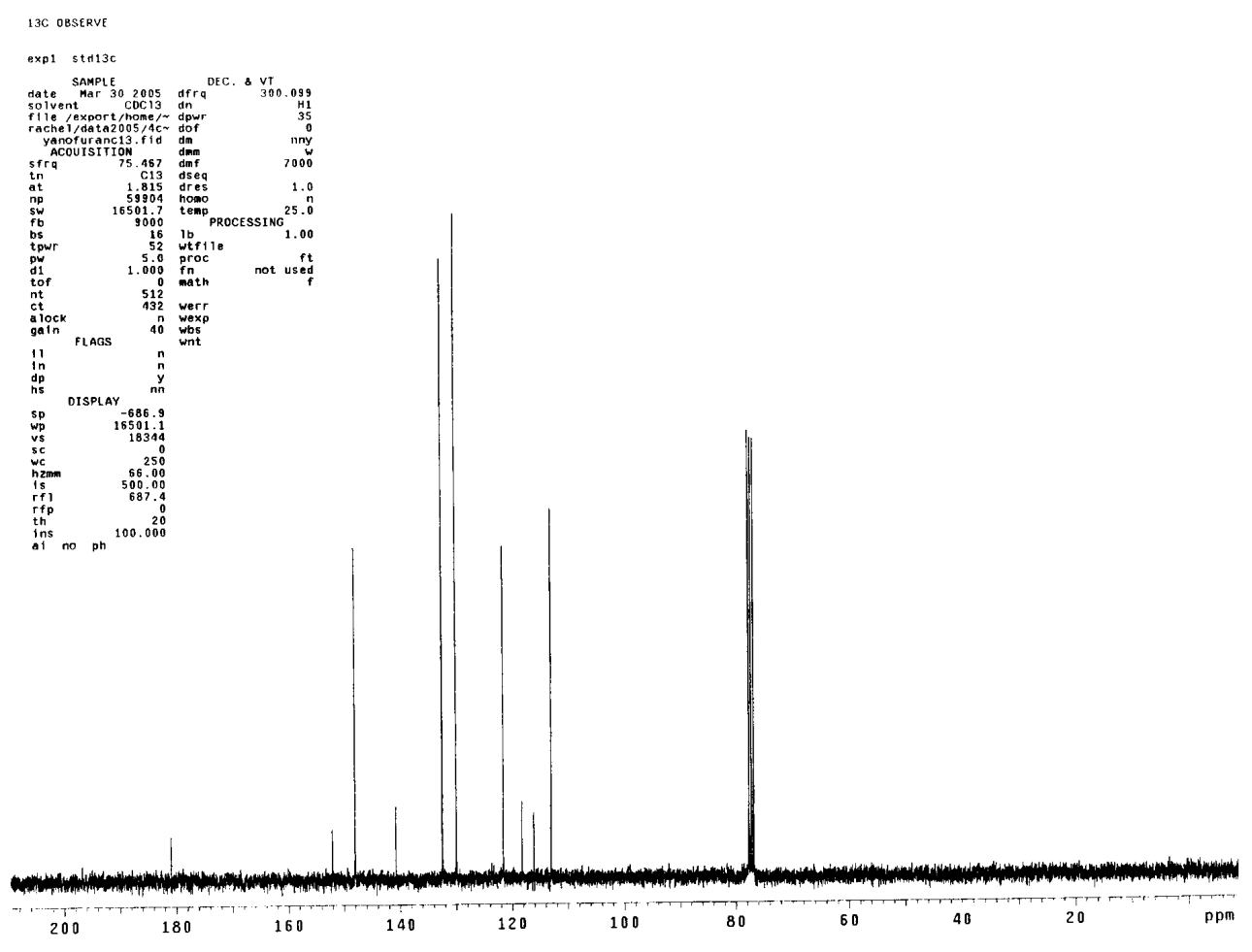

${ }^{1}$ H NMR and ${ }^{13}$ NMR spectra of 4-Cyanophenyl phenylacetylene ketone (11).
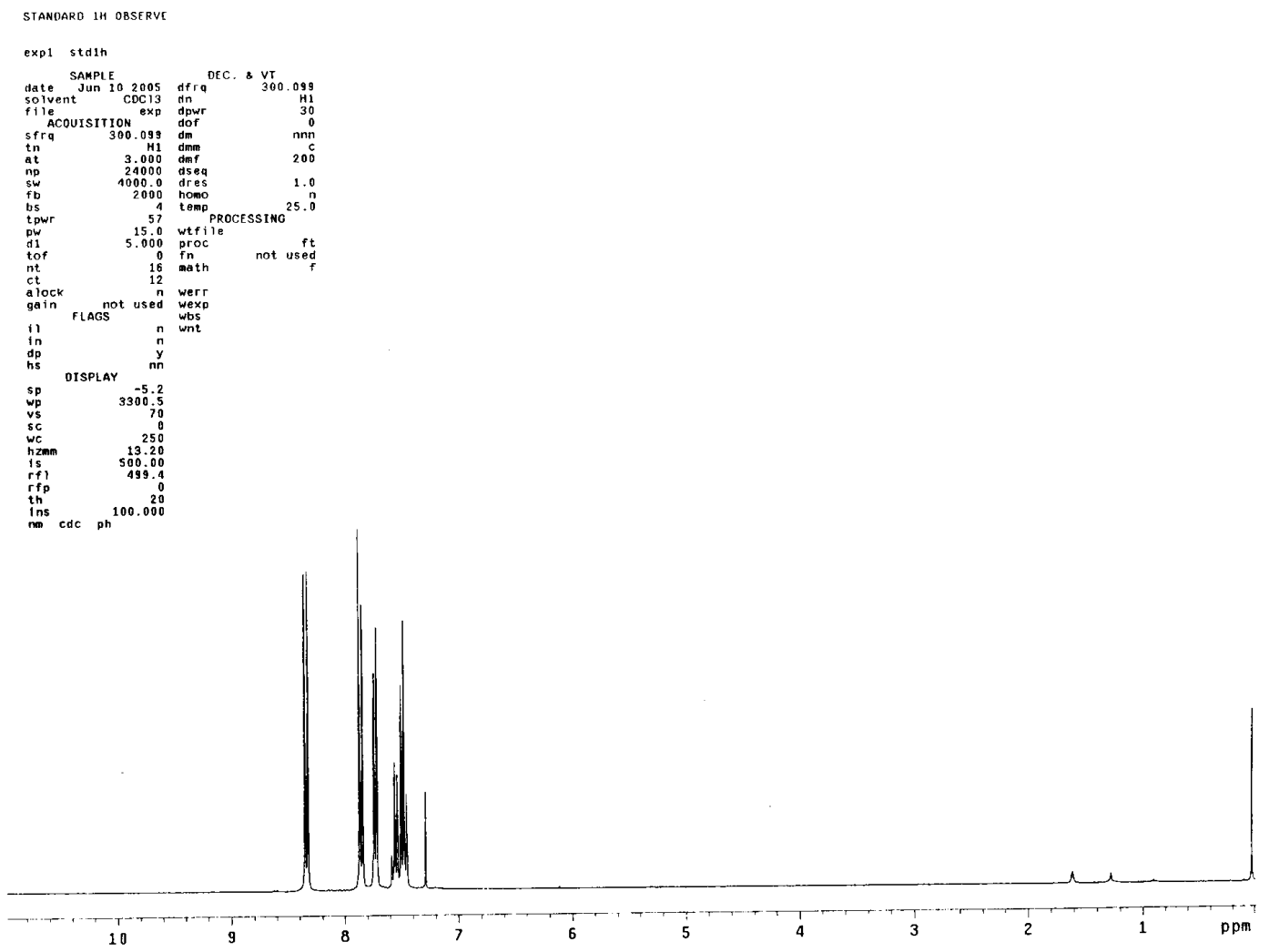


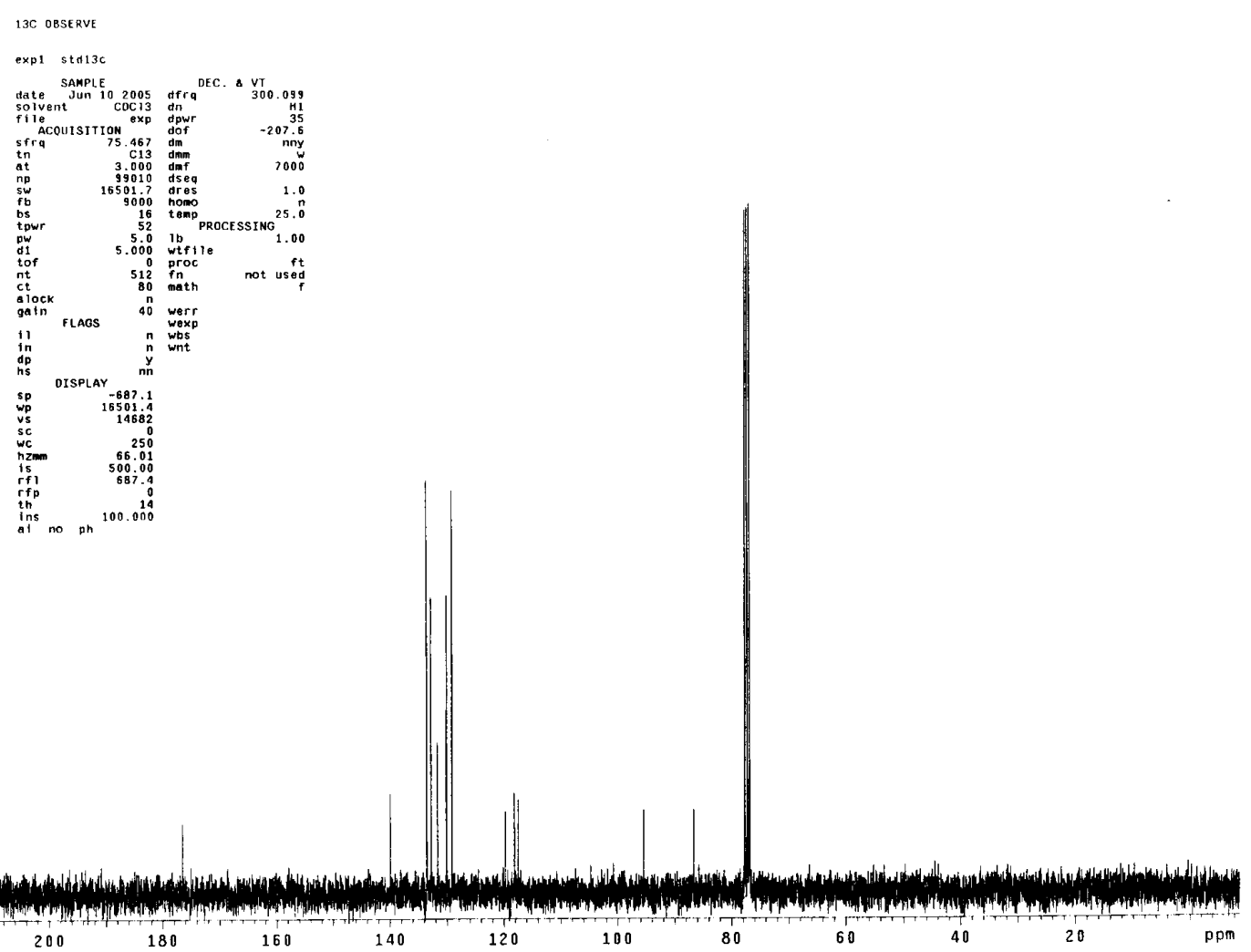

\section{${ }^{1}$ H NMR and ${ }^{13}$ NMR spectra of 2-Furan 4-methoxyphenyl ketone (13).}
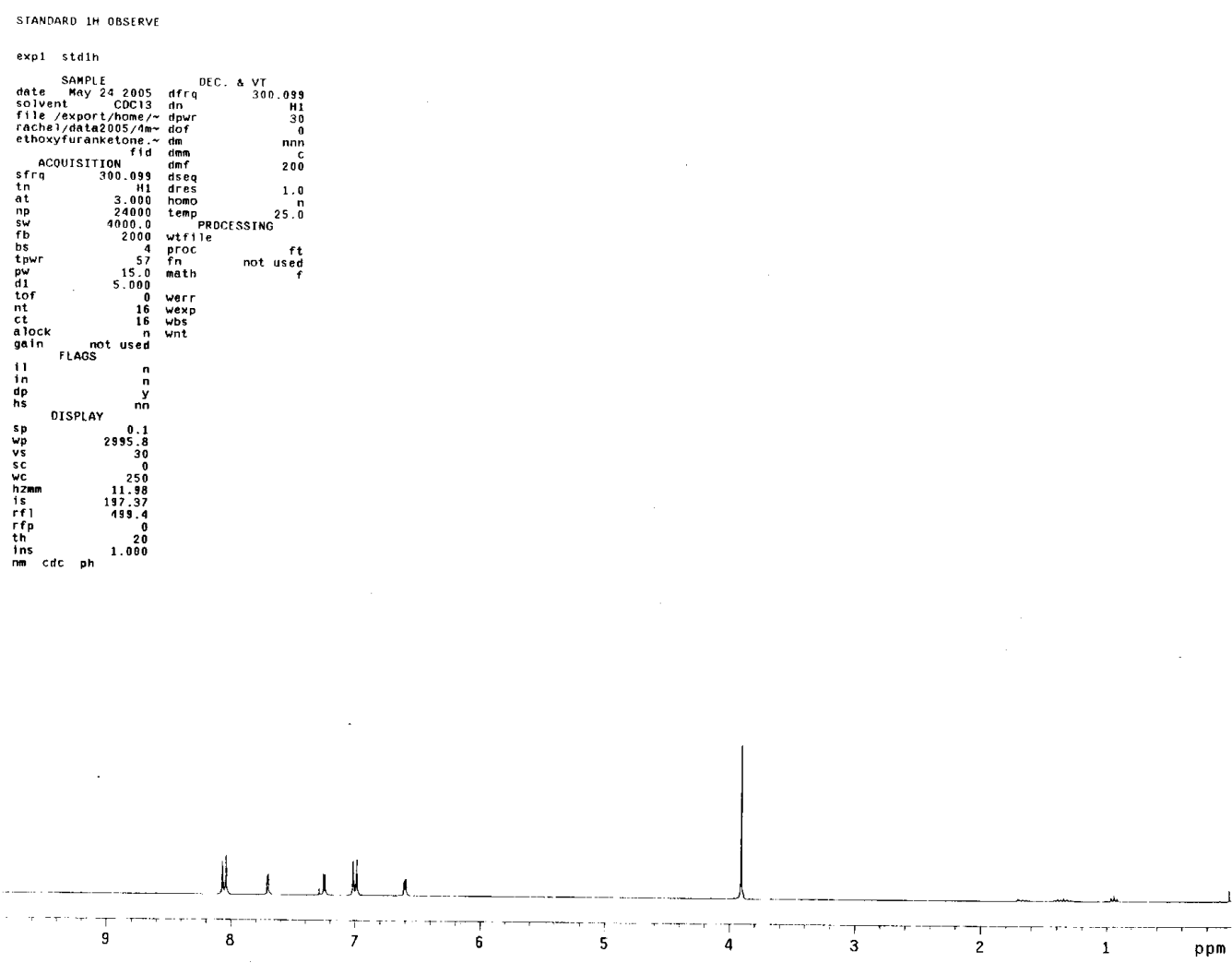

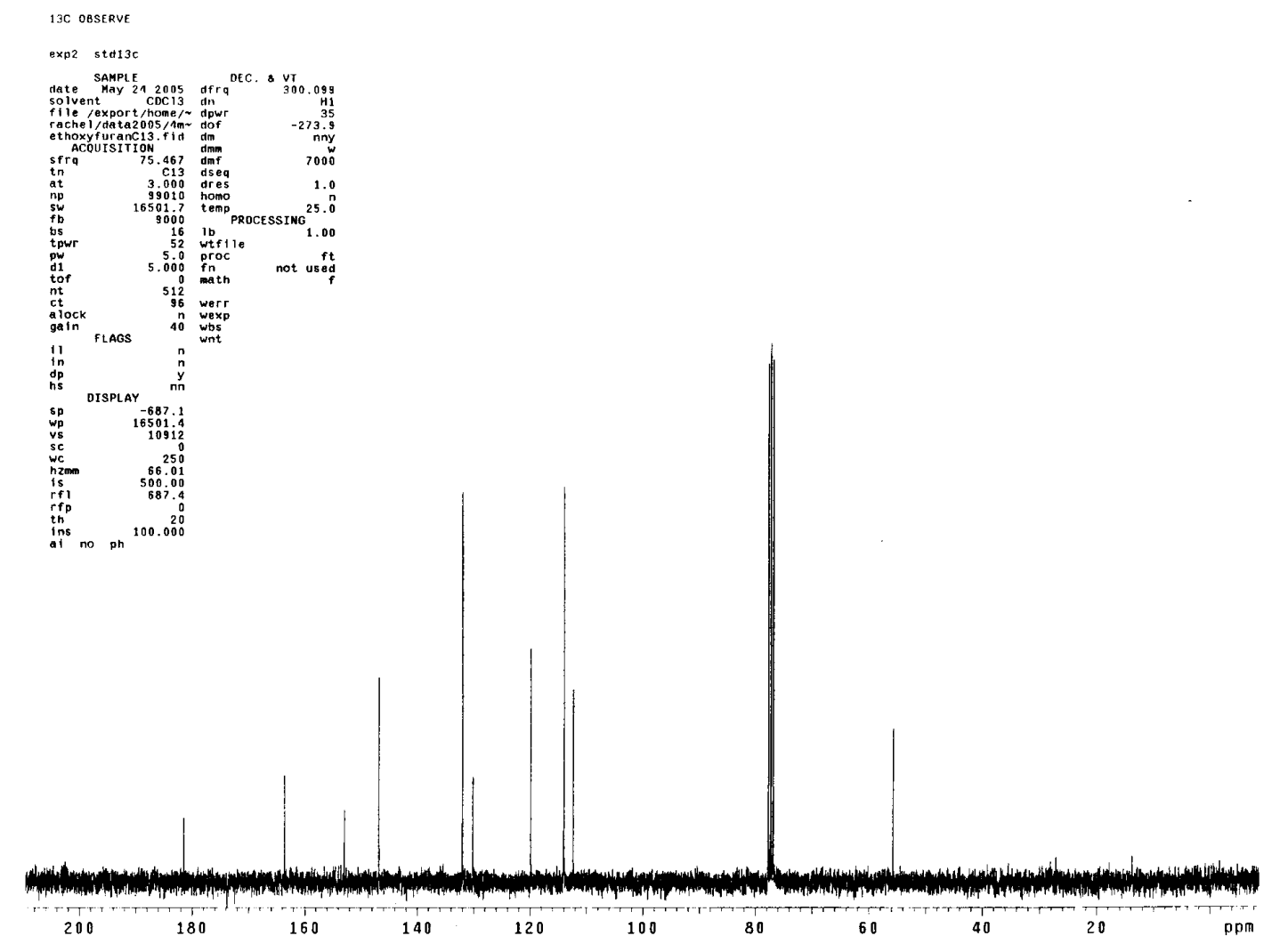

${ }^{1}$ H NMR and ${ }^{13}$ NMR spectra of 2-Furan piperonyloyl ketone (15).
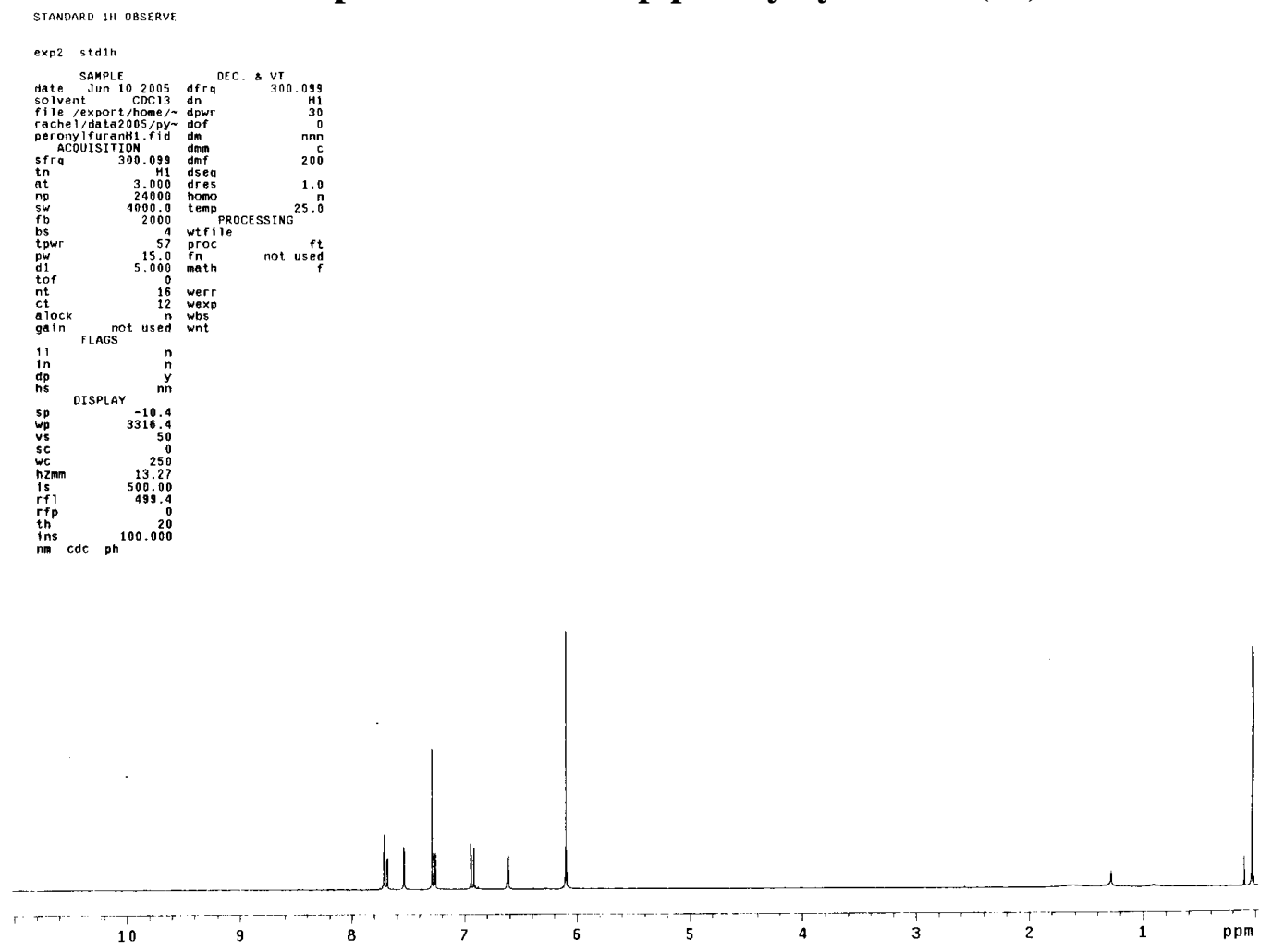


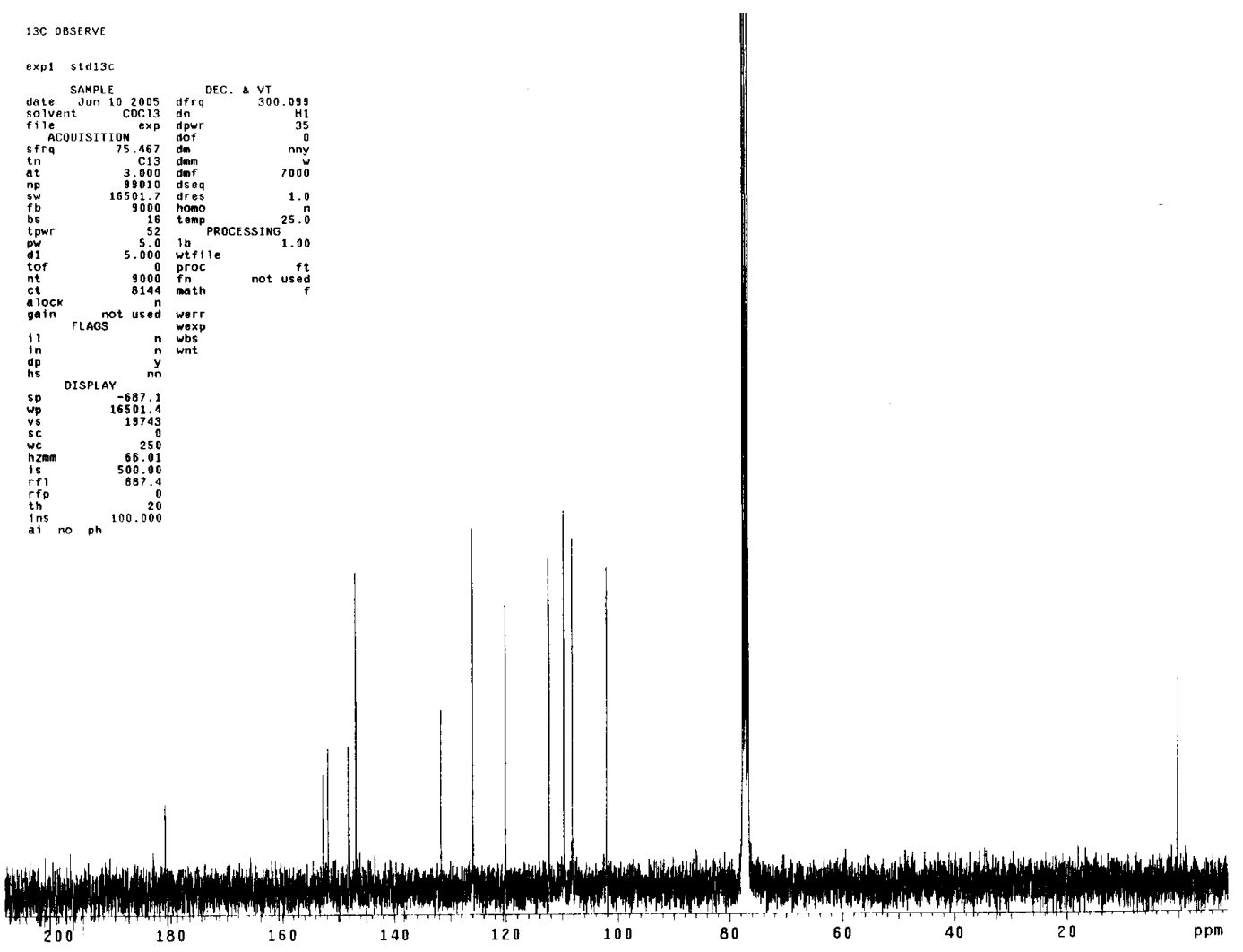

${ }^{1} \mathrm{H}$ NMR and ${ }^{13}$ NMR spectra of Piperonyloyl 2-thiophen ketone (17).
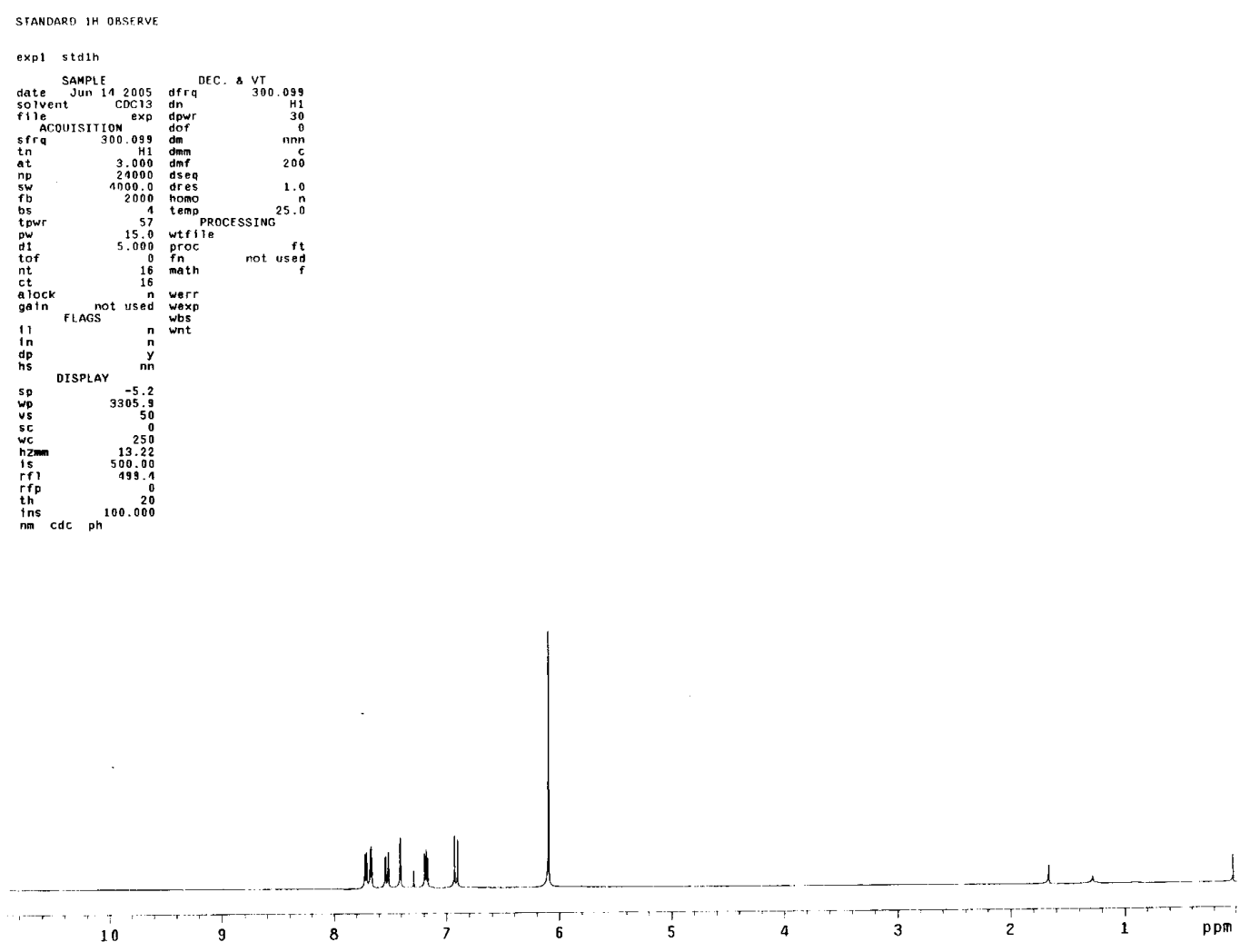


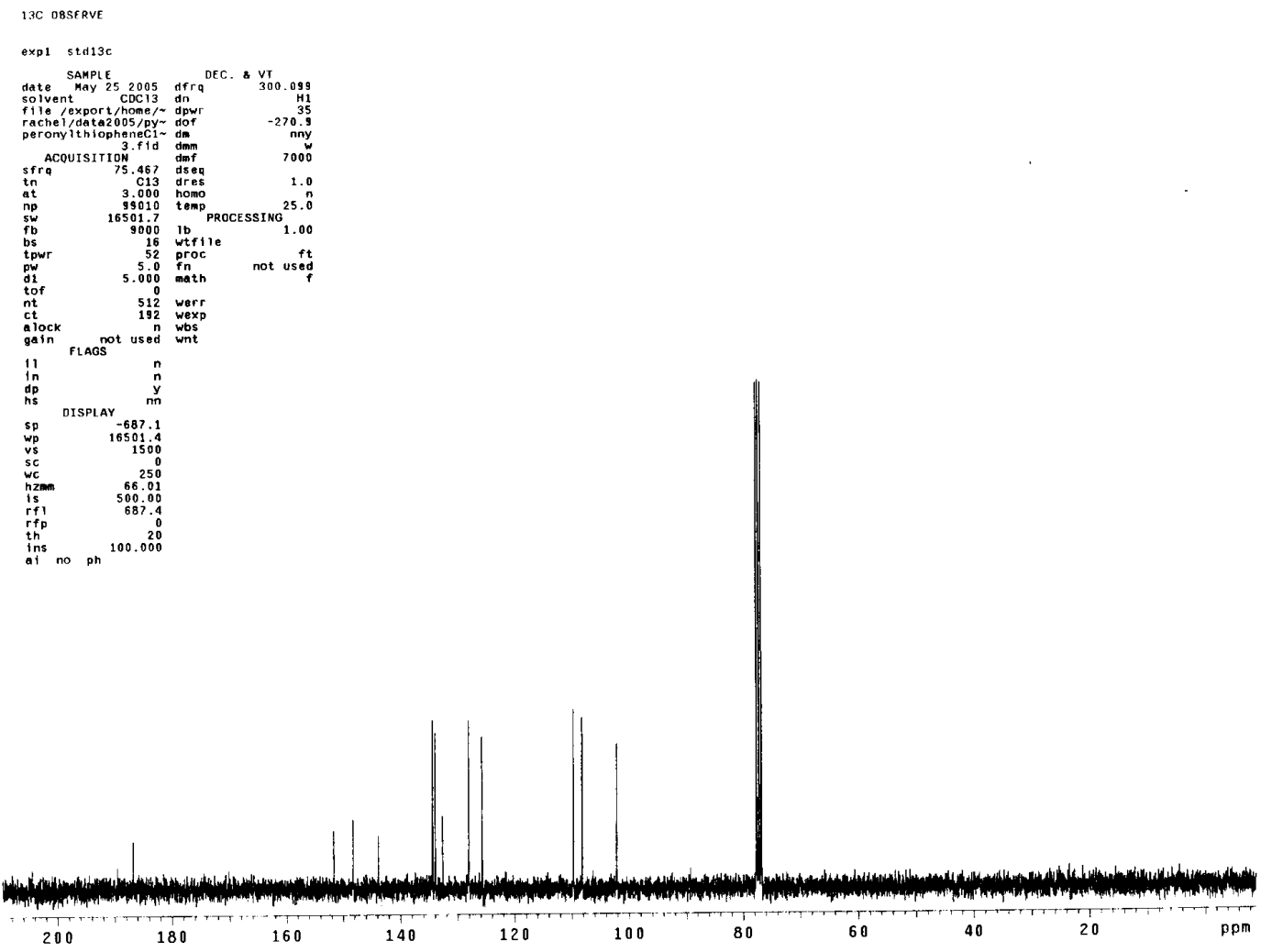

${ }^{1}$ H NMR and ${ }^{13}$ NMR spectra of Phenylacetylene piperonyloyl ketone (18).
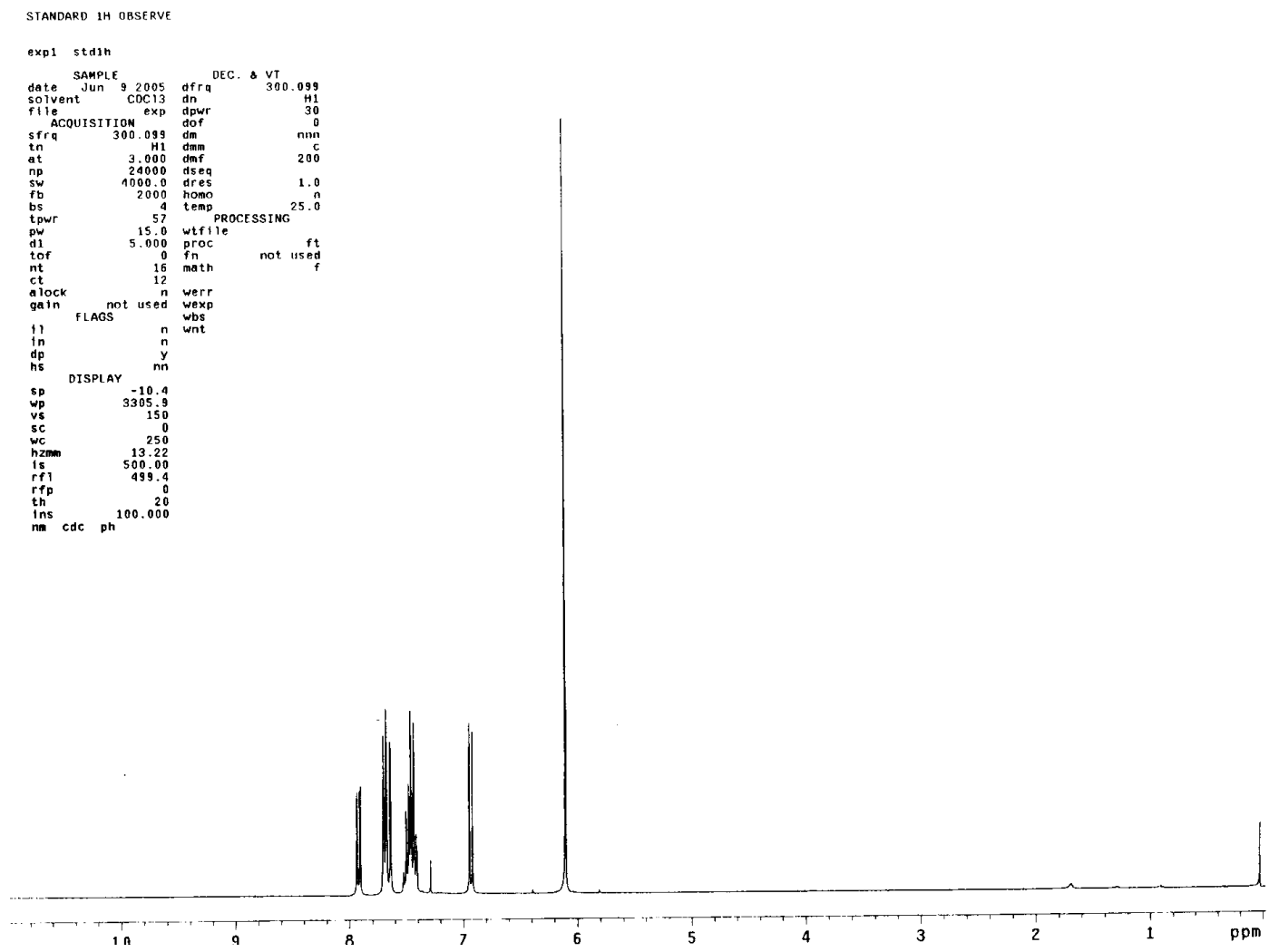


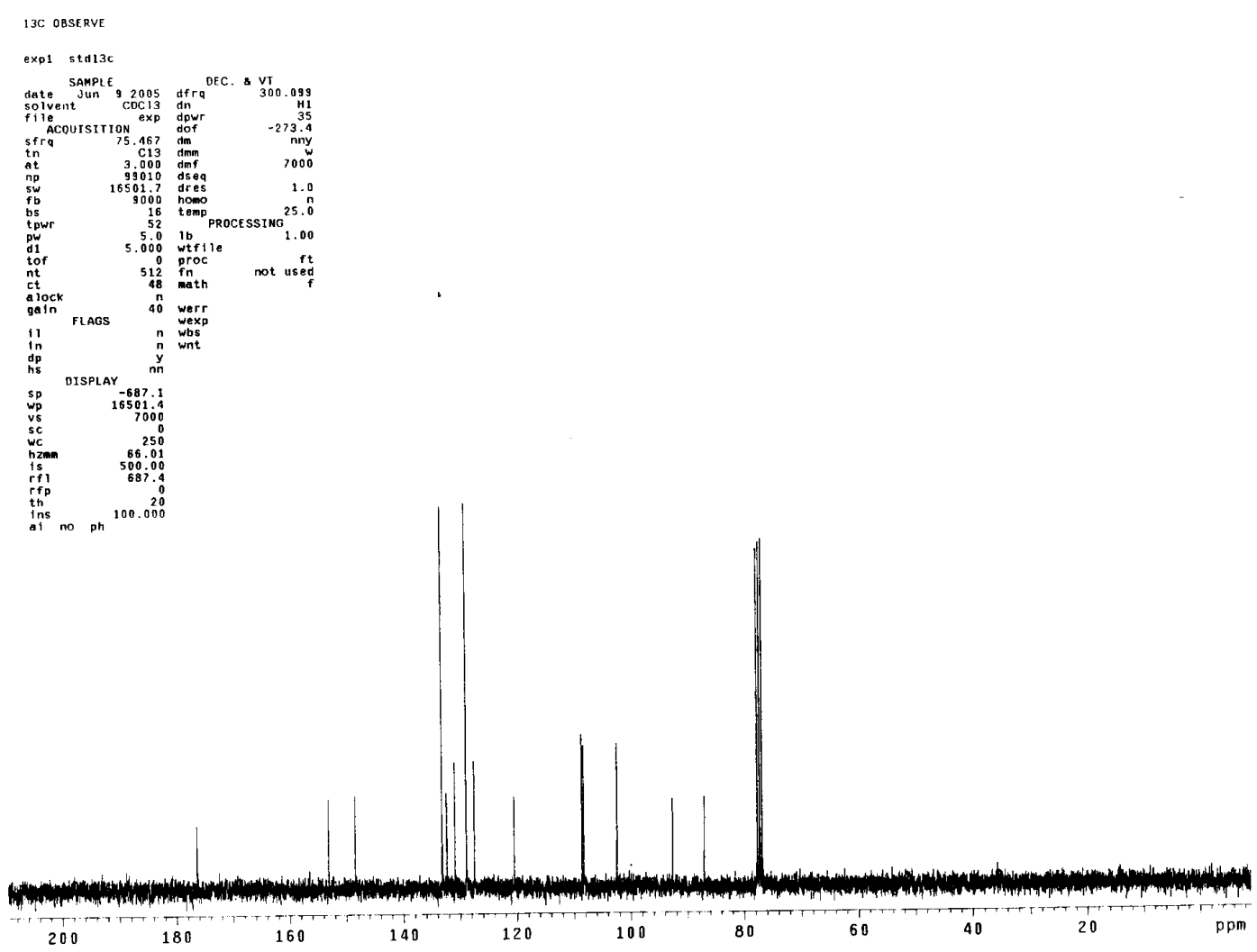

${ }^{1}$ H NMR and ${ }^{13}$ NMR spectra of Propyl 2-thiophen ketone (20).
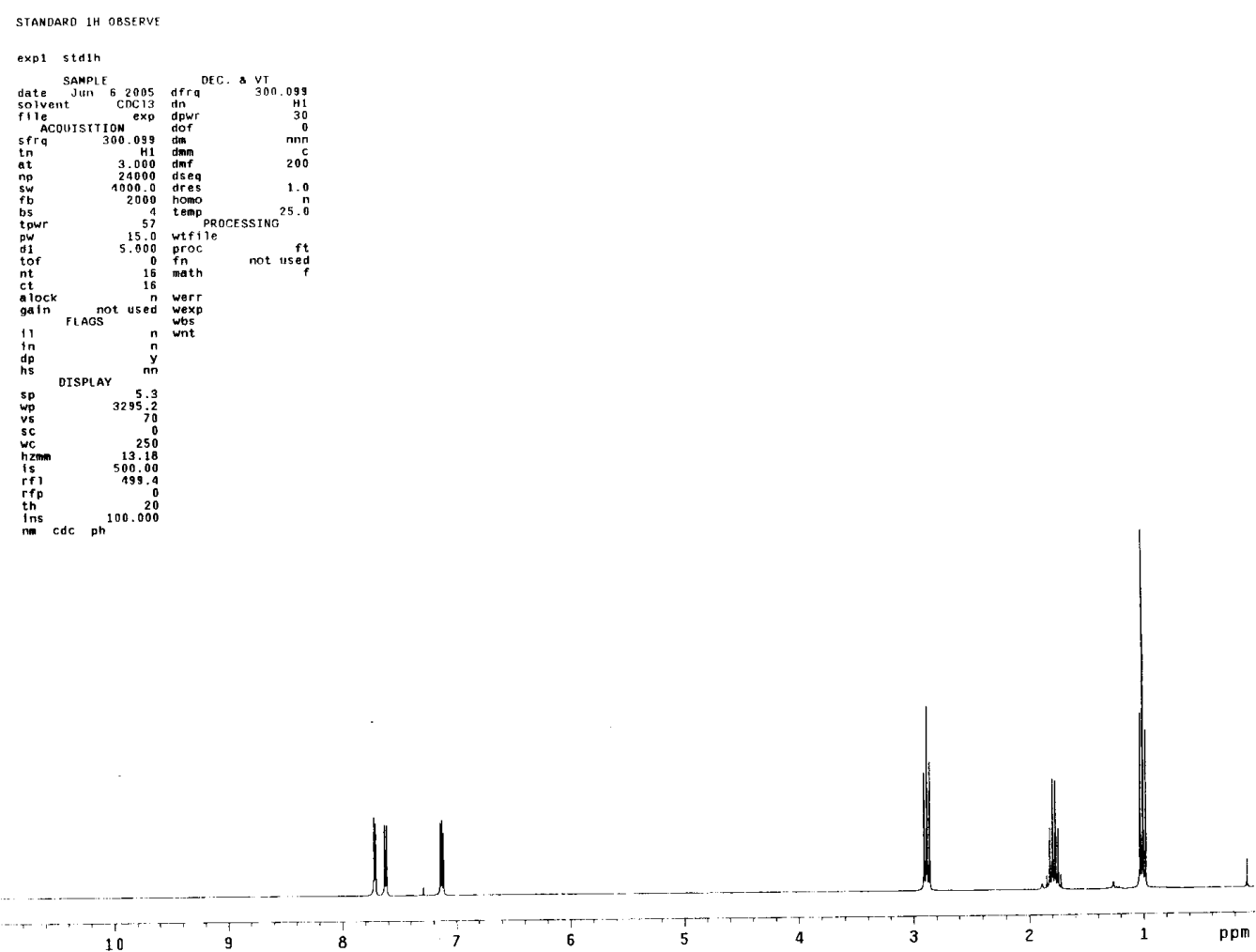


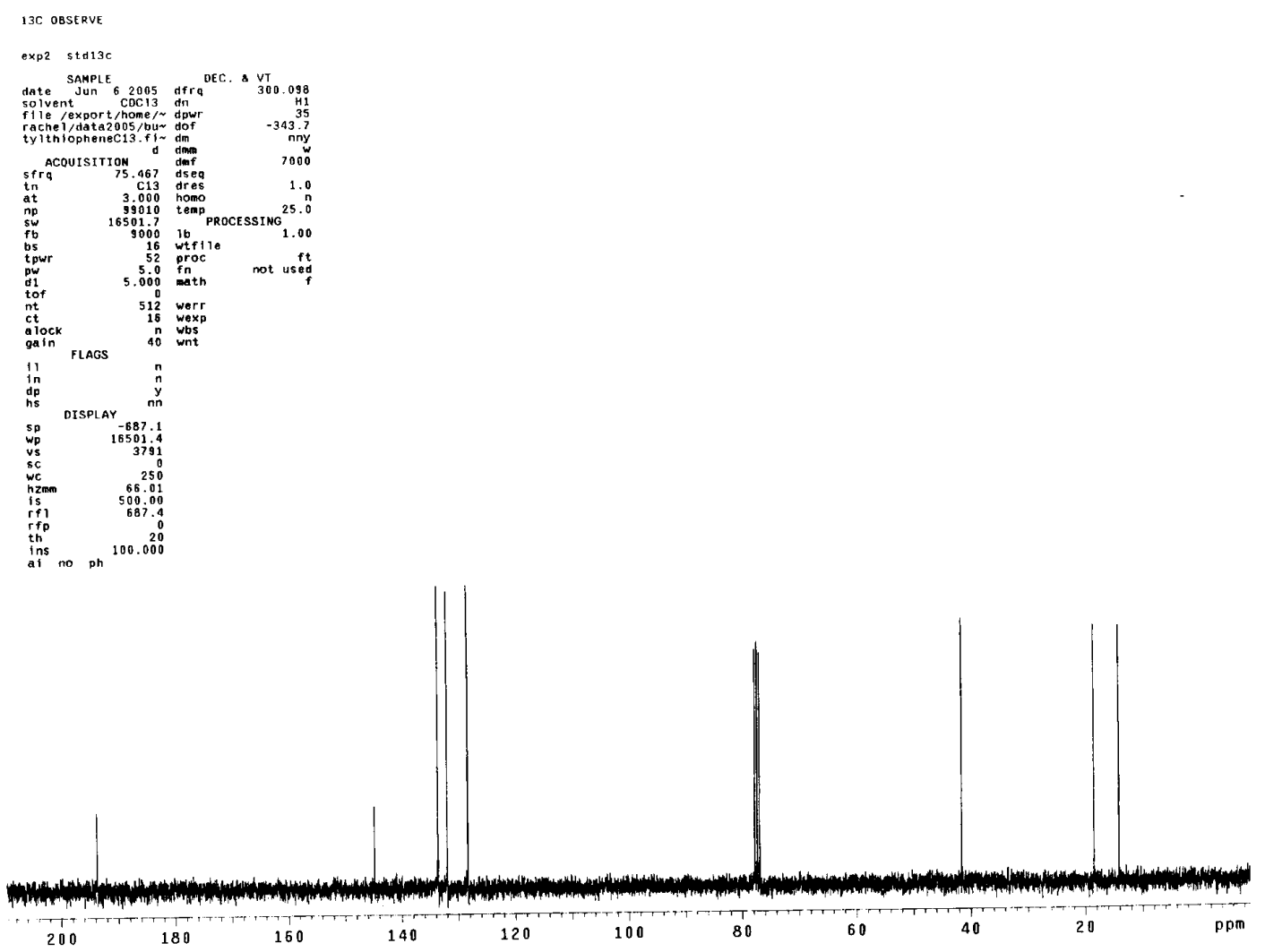

${ }^{1}$ H NMR and ${ }^{13}$ NMR spectra of 2-Pivaloylfuran (22).
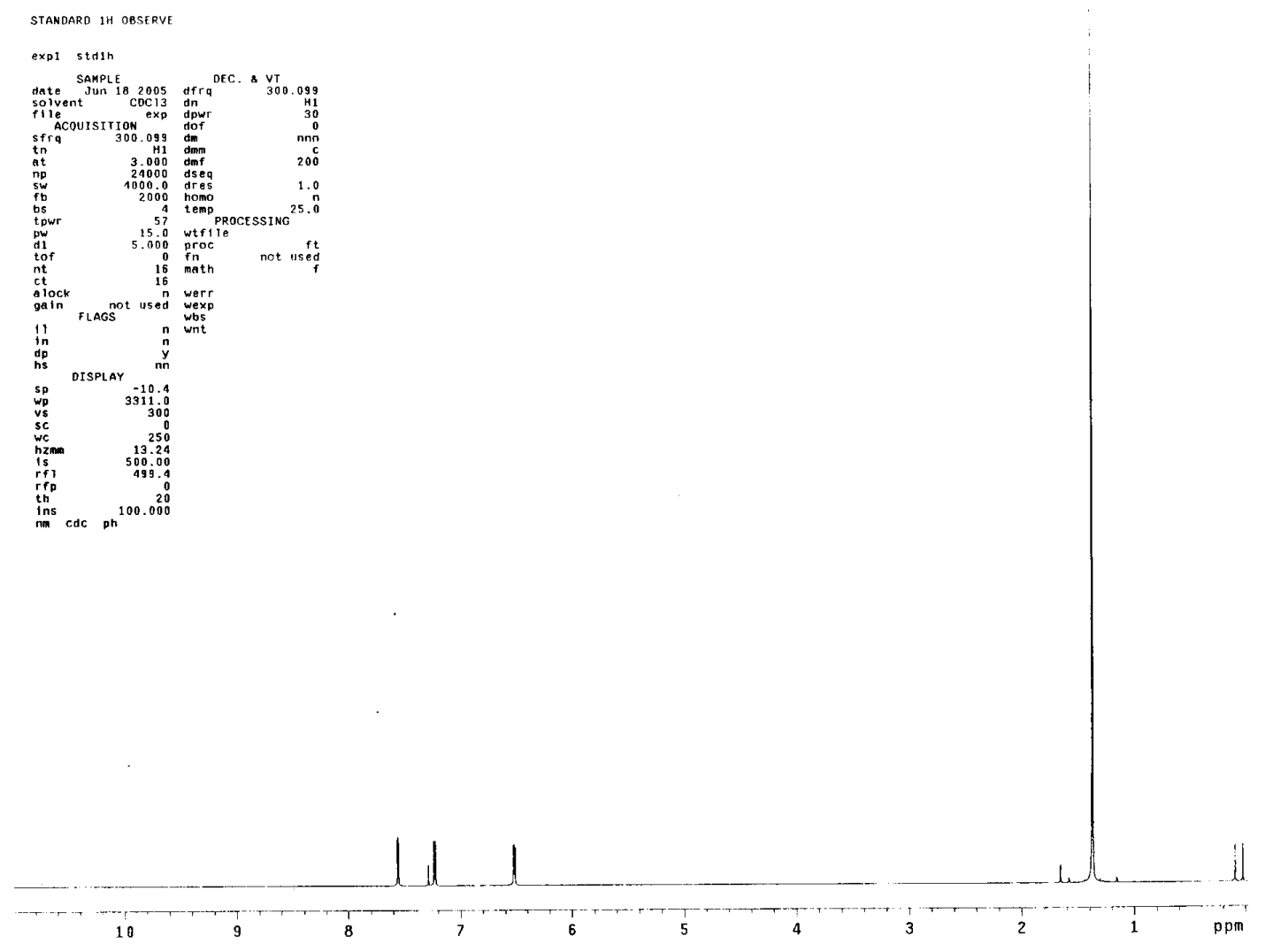

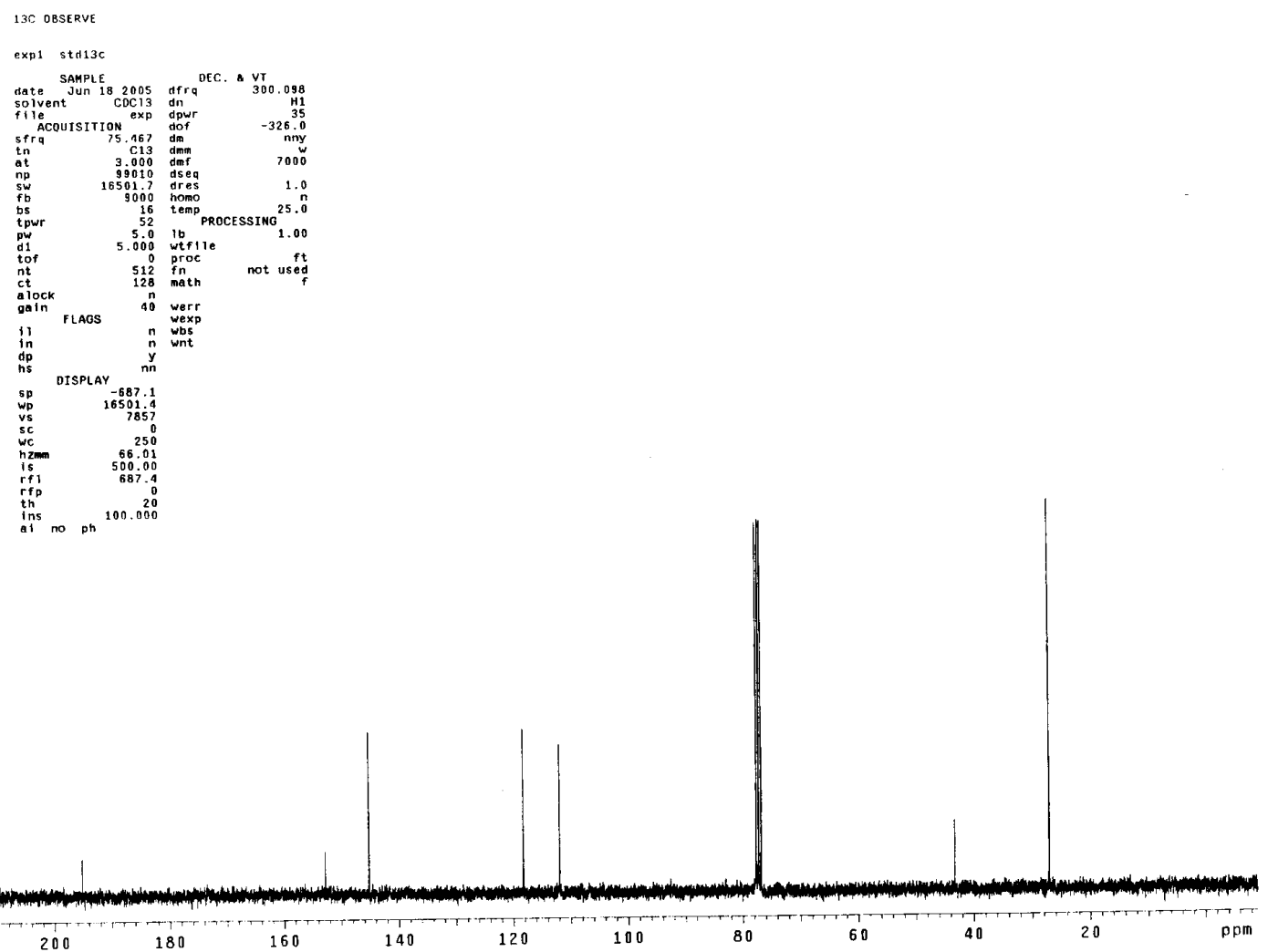

${ }^{1} \mathrm{H}$ NMR and ${ }^{13}$ NMR spectra of 4-Chlorophenyl 2-furan ketone (24).
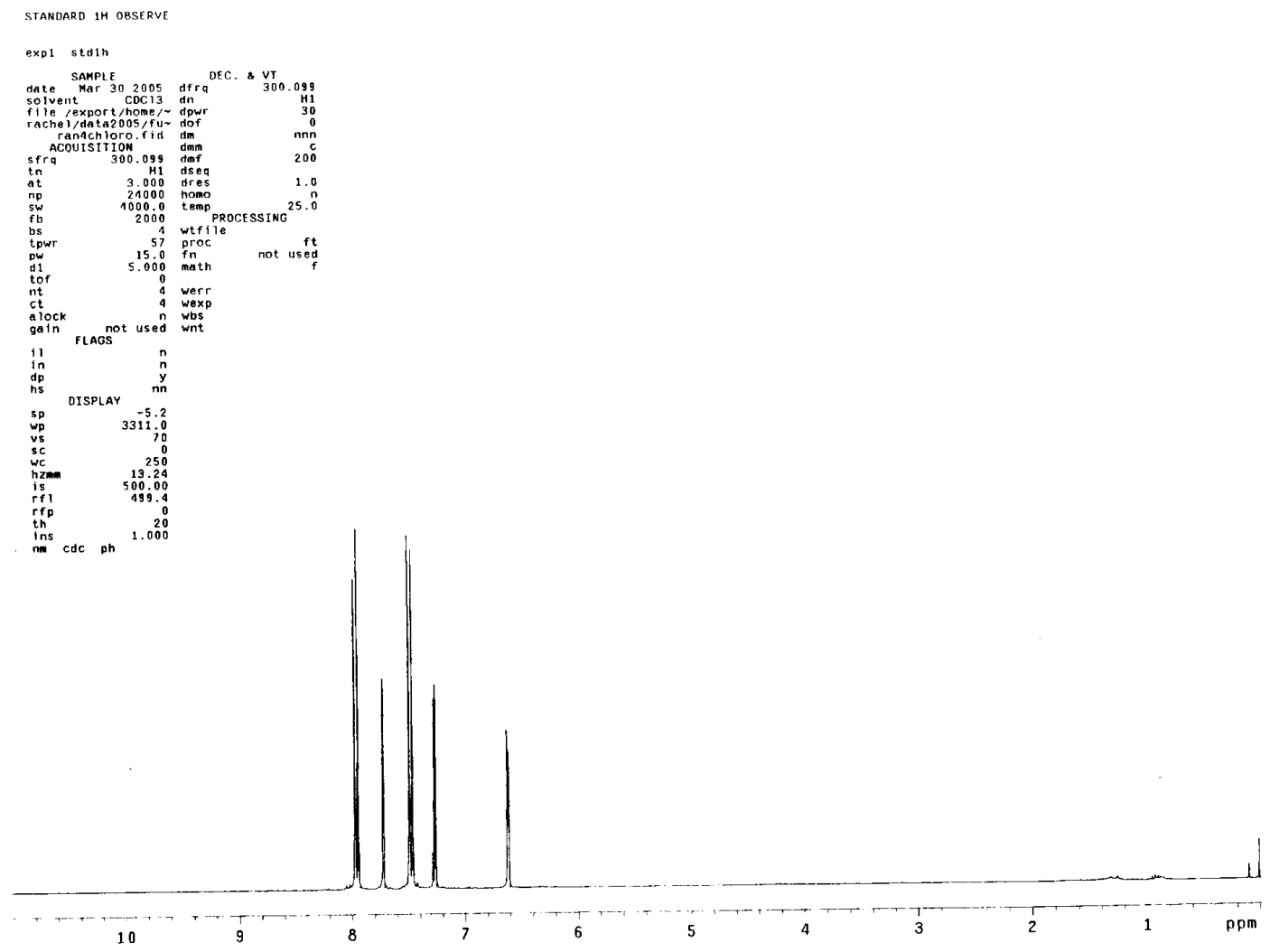


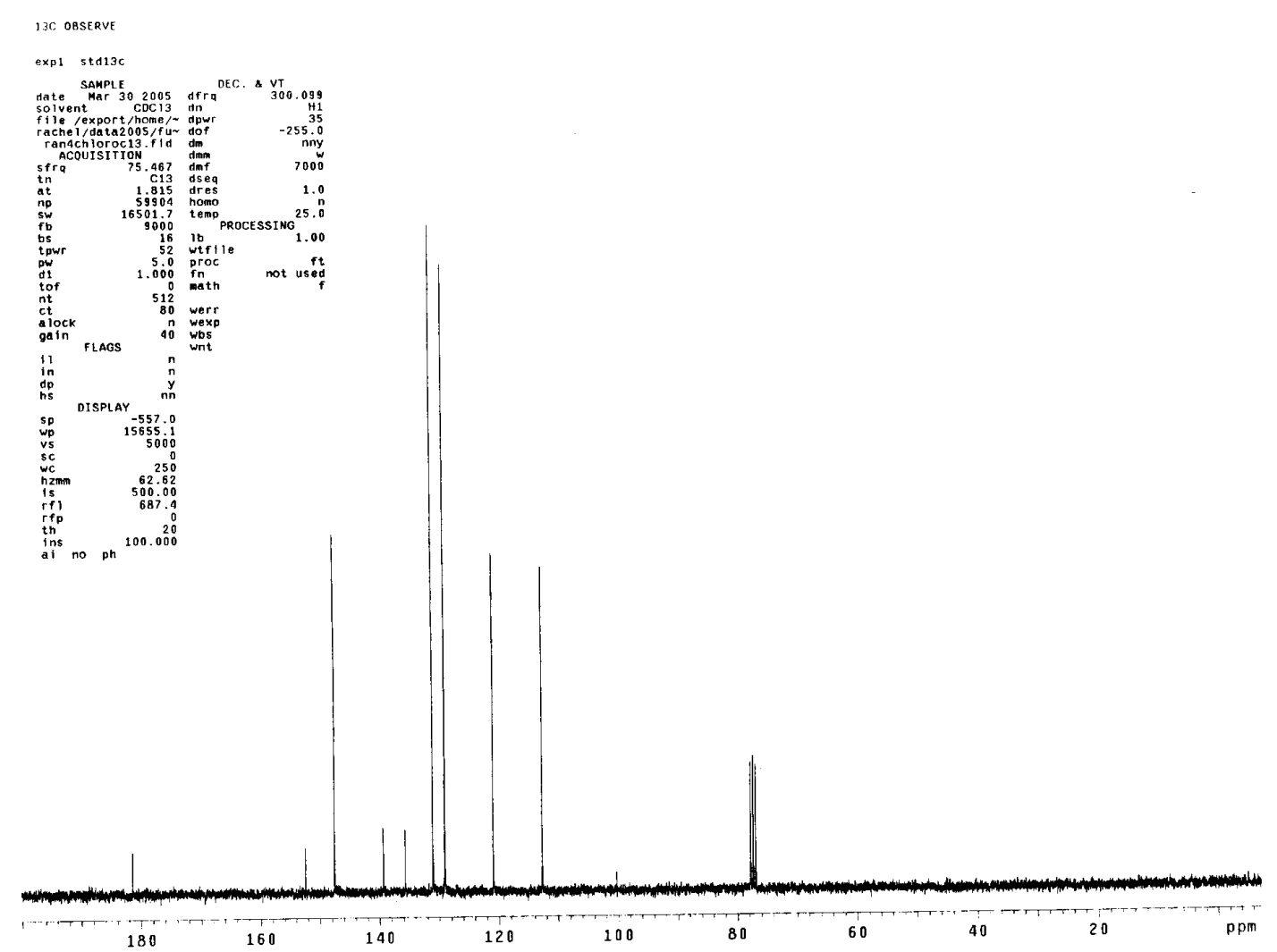

${ }^{1} \mathrm{H}$ NMR and ${ }^{13}$ NMR spectra of 3-Chlorophenyl 2-furan ketone (28).

Standard 1 H OBSERVE

exp1 stdin
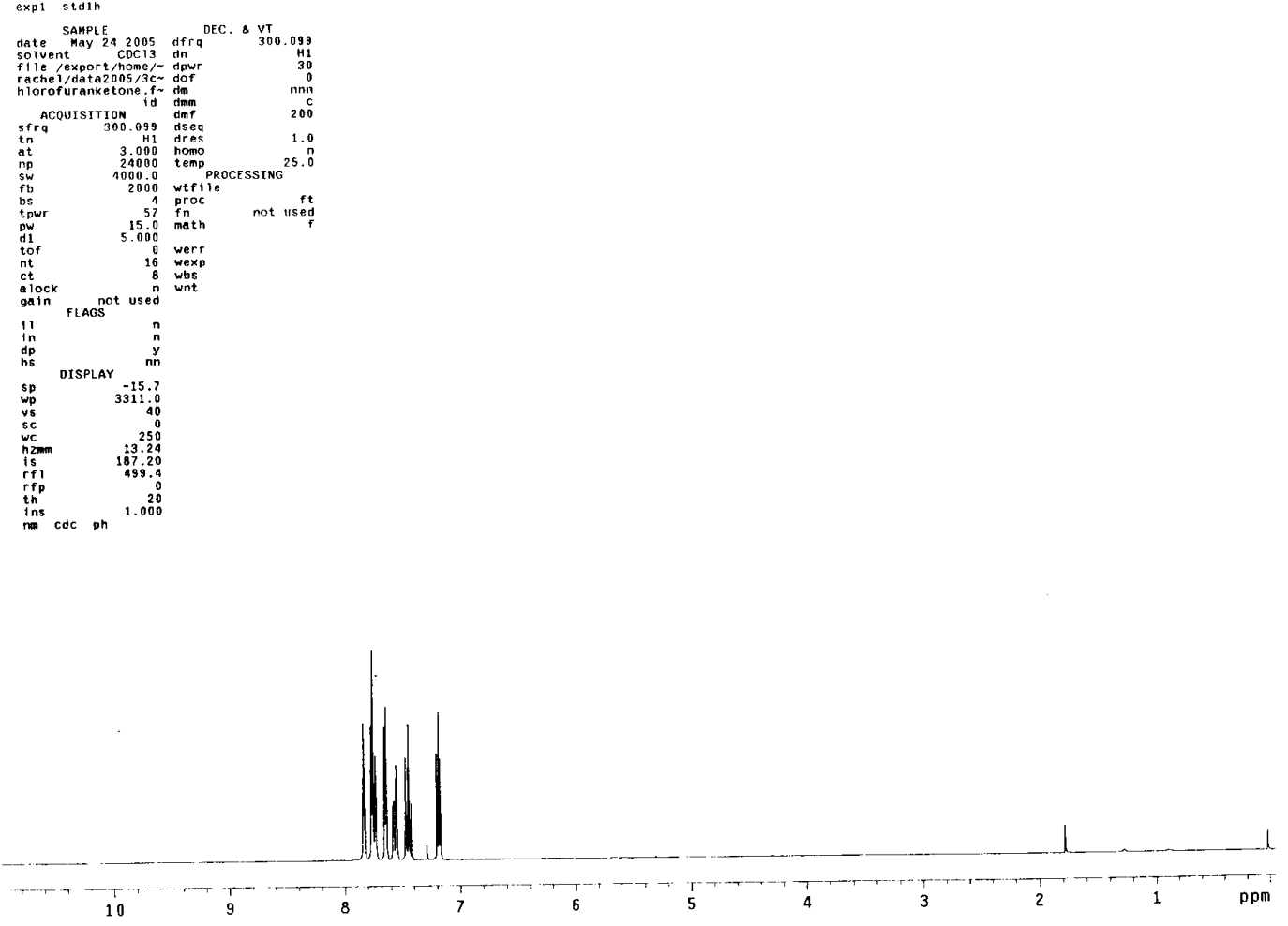

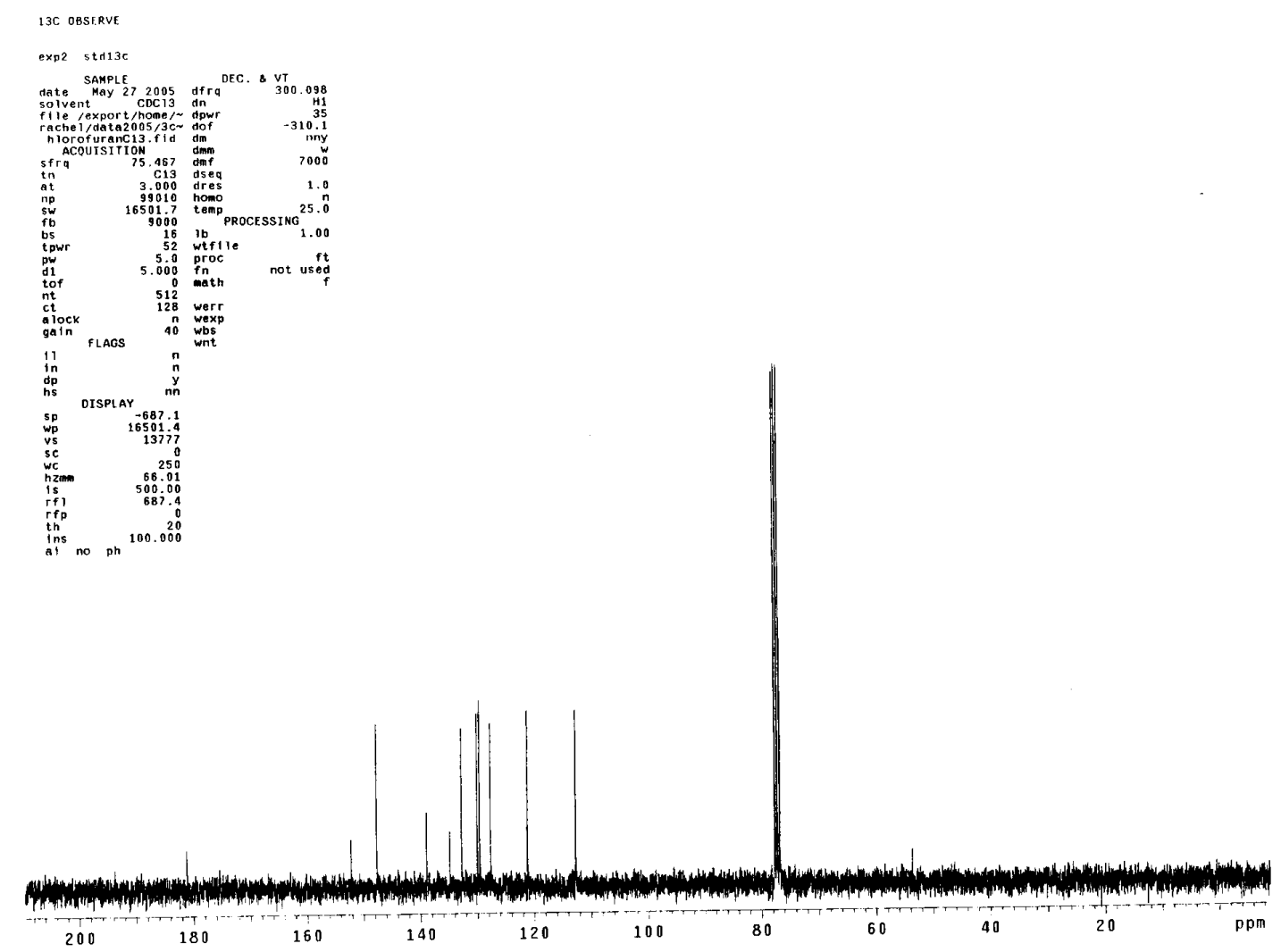

${ }^{1}$ H NMR and ${ }^{13}$ NMR spectra of 2-Chlorophenyl 2-furan ketone (30).
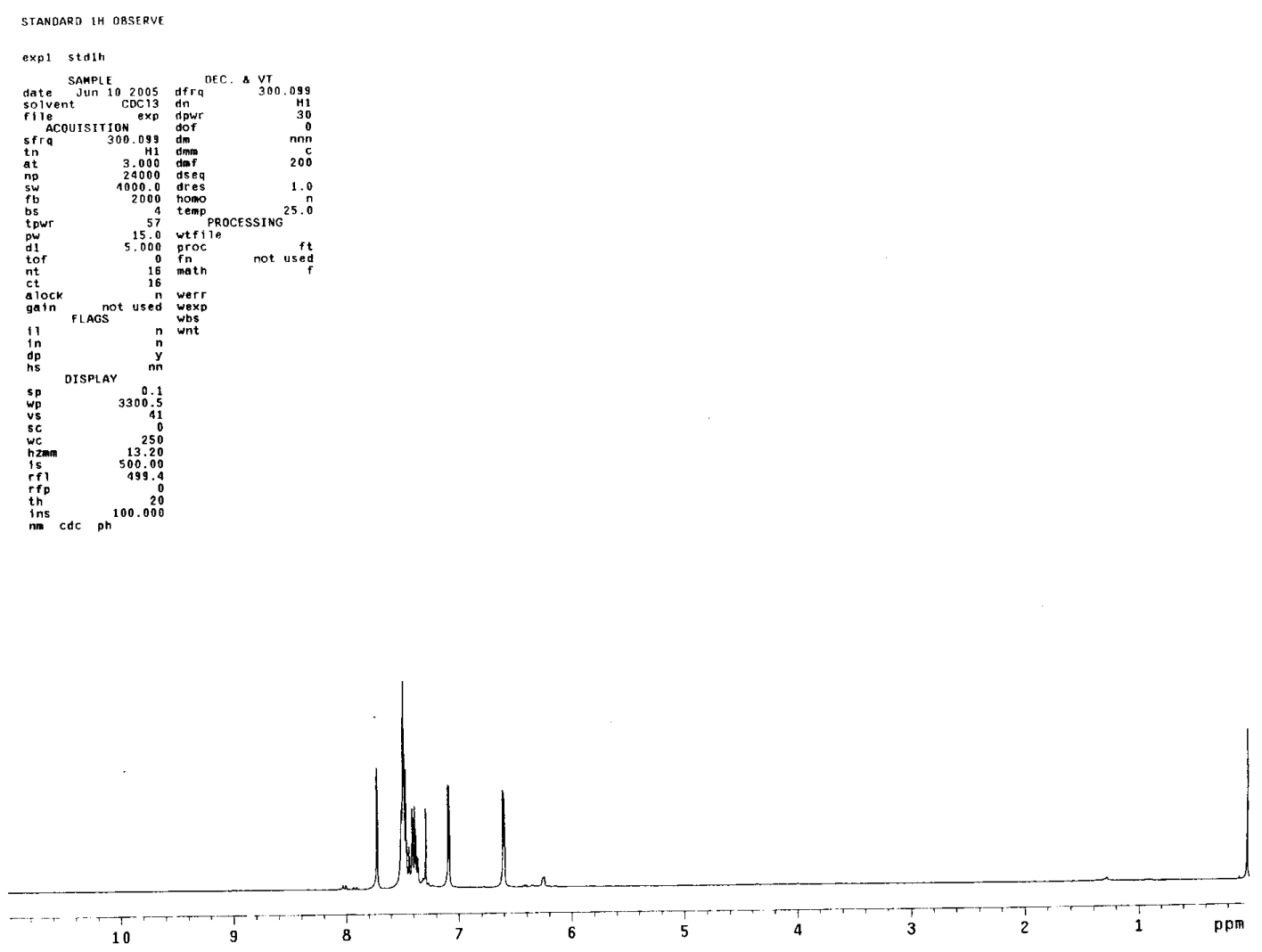

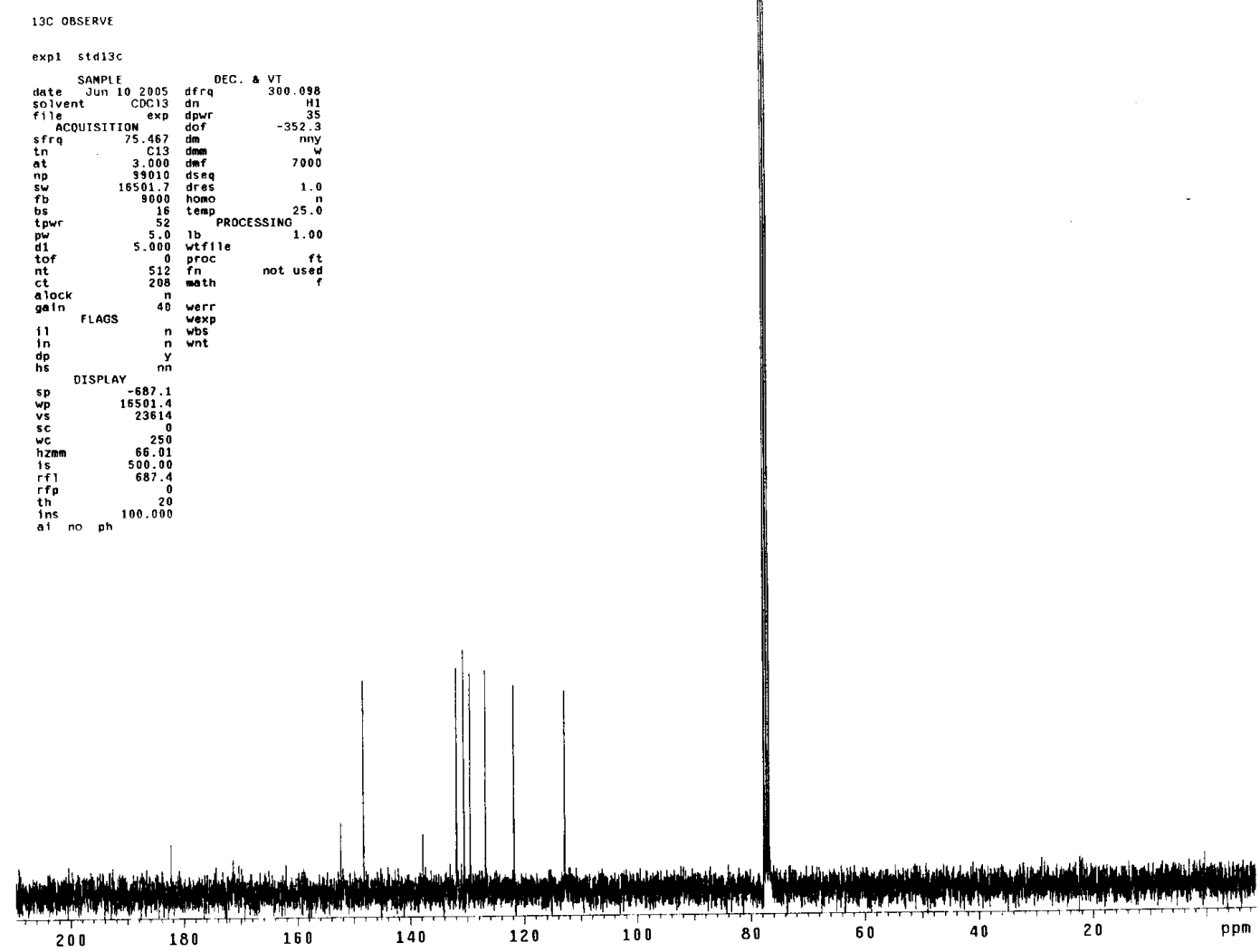

${ }^{1}$ H NMR and ${ }^{13}$ NMR spectra of 4-Chlorophenyl phenylacetylene ketone (31).
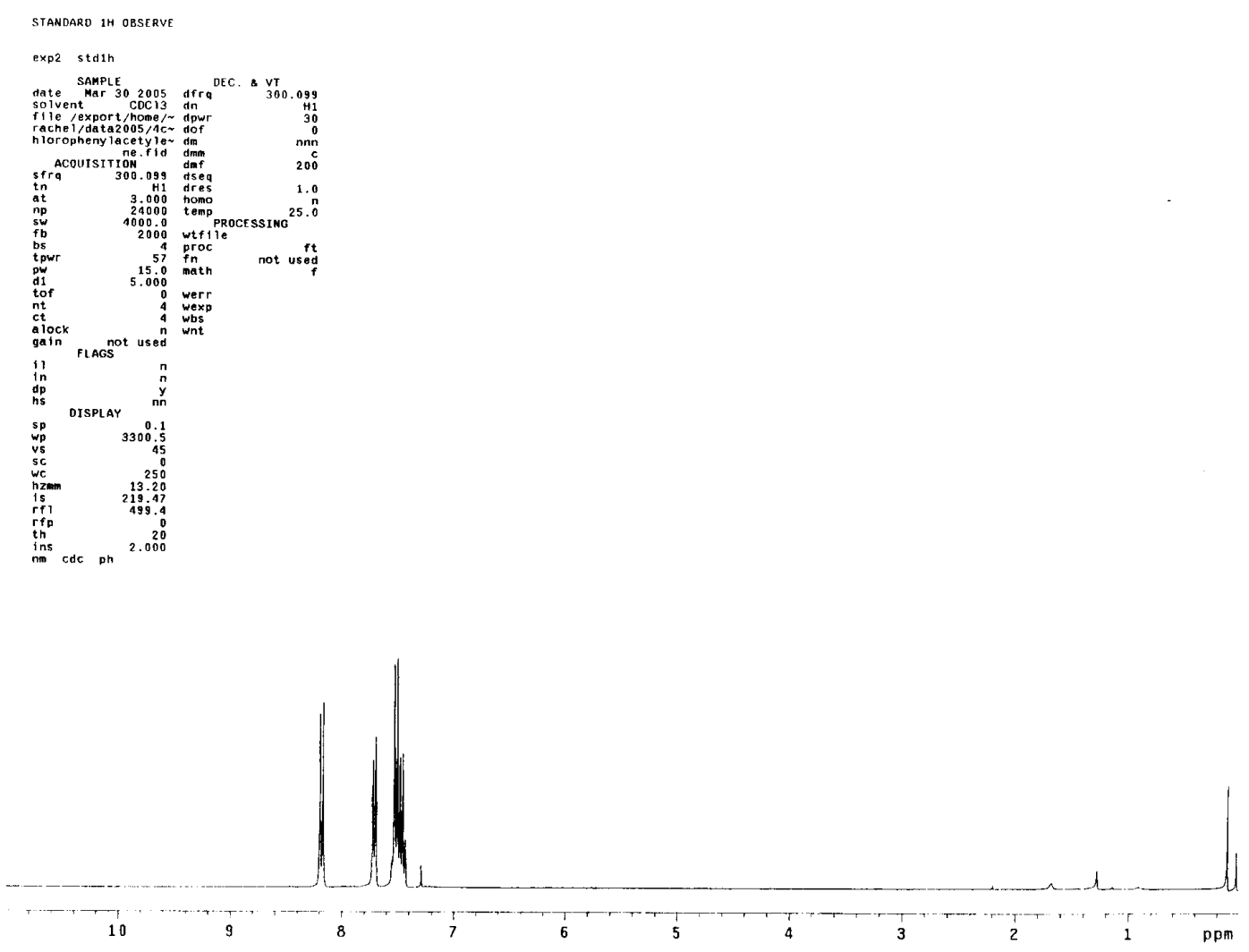


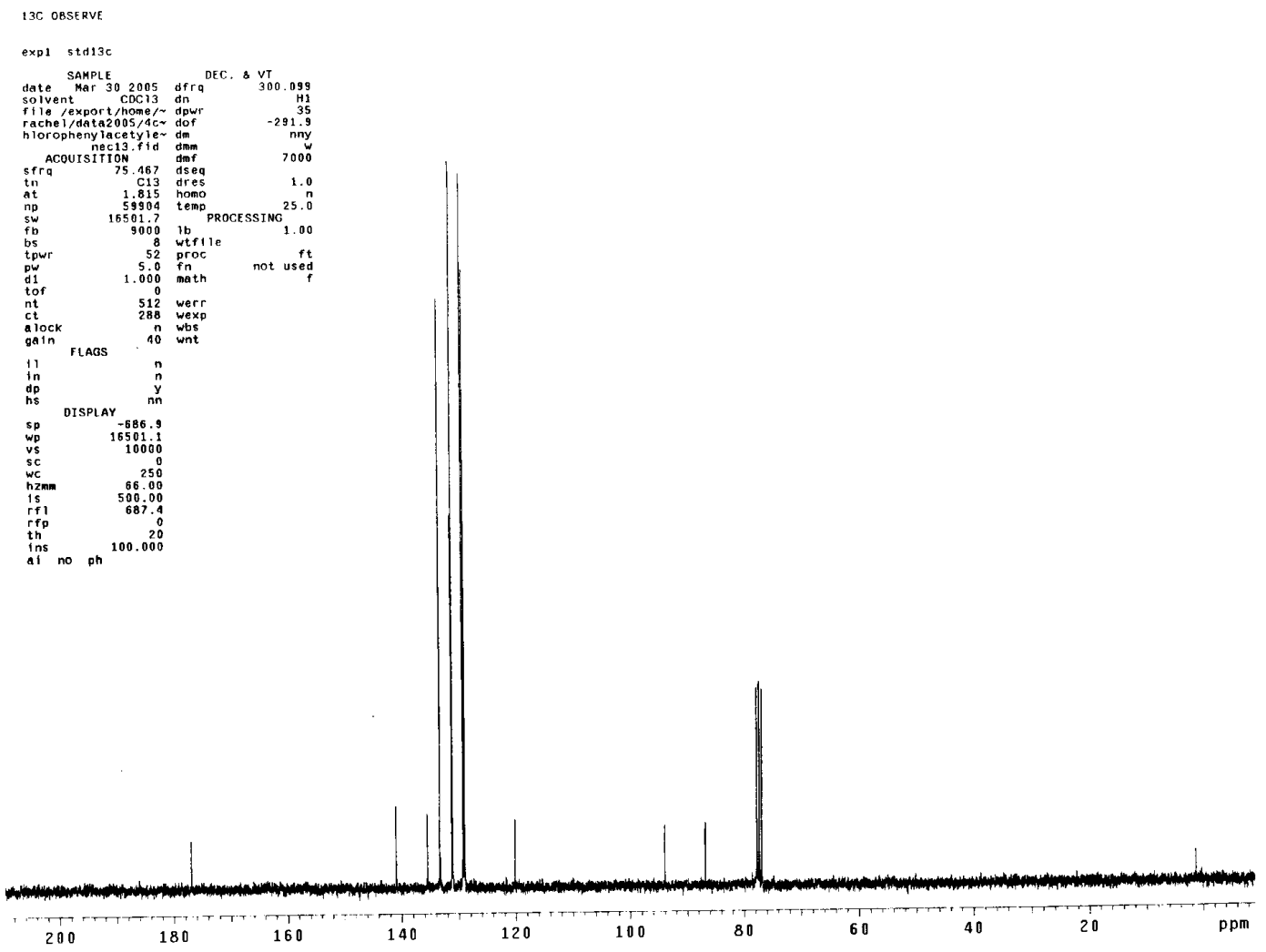

${ }^{1} \mathrm{H}$ NMR and ${ }^{13}$ NMR spectra of 4-Chlorophenyl 2-thiophen ketone (32).
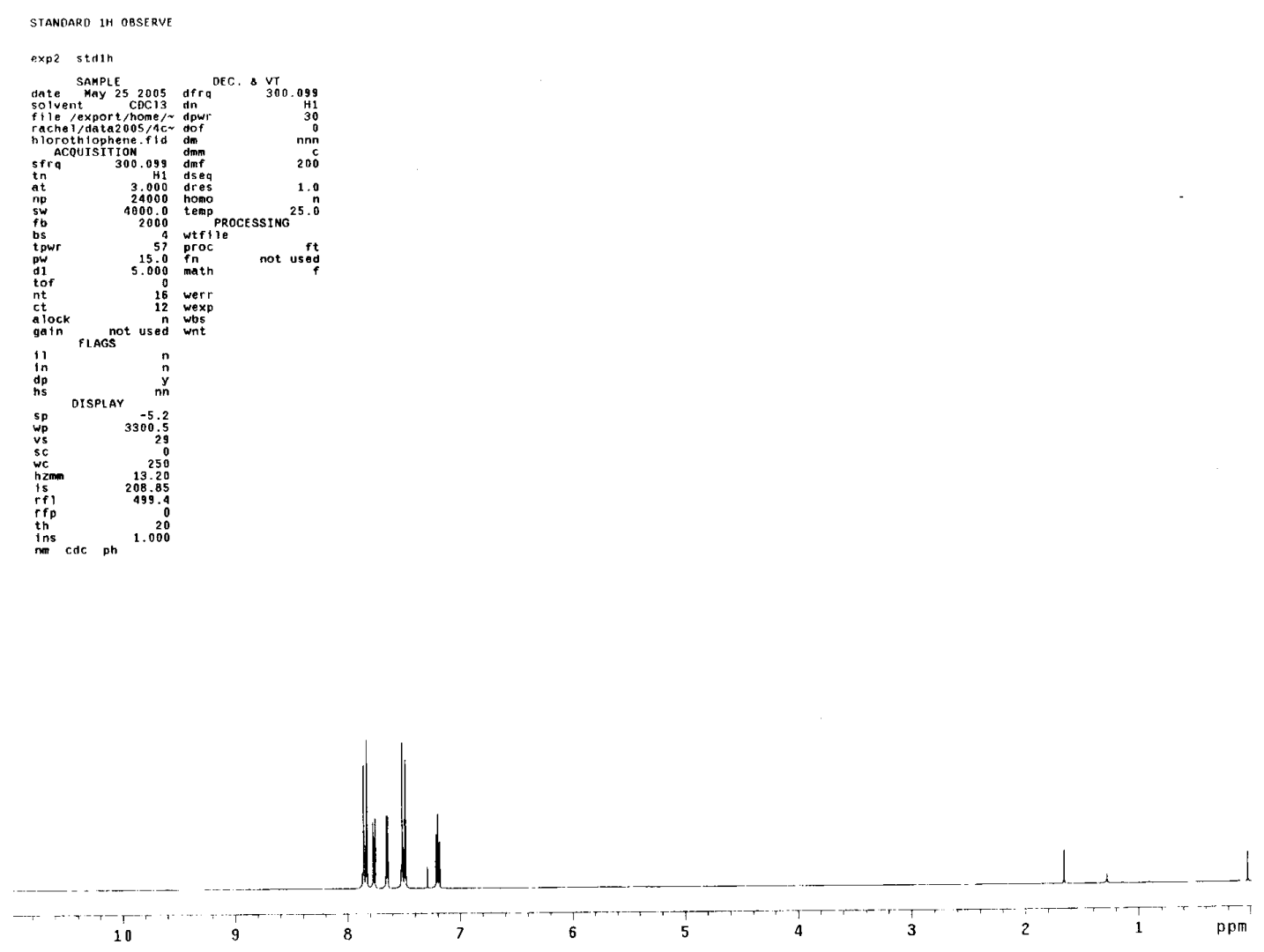

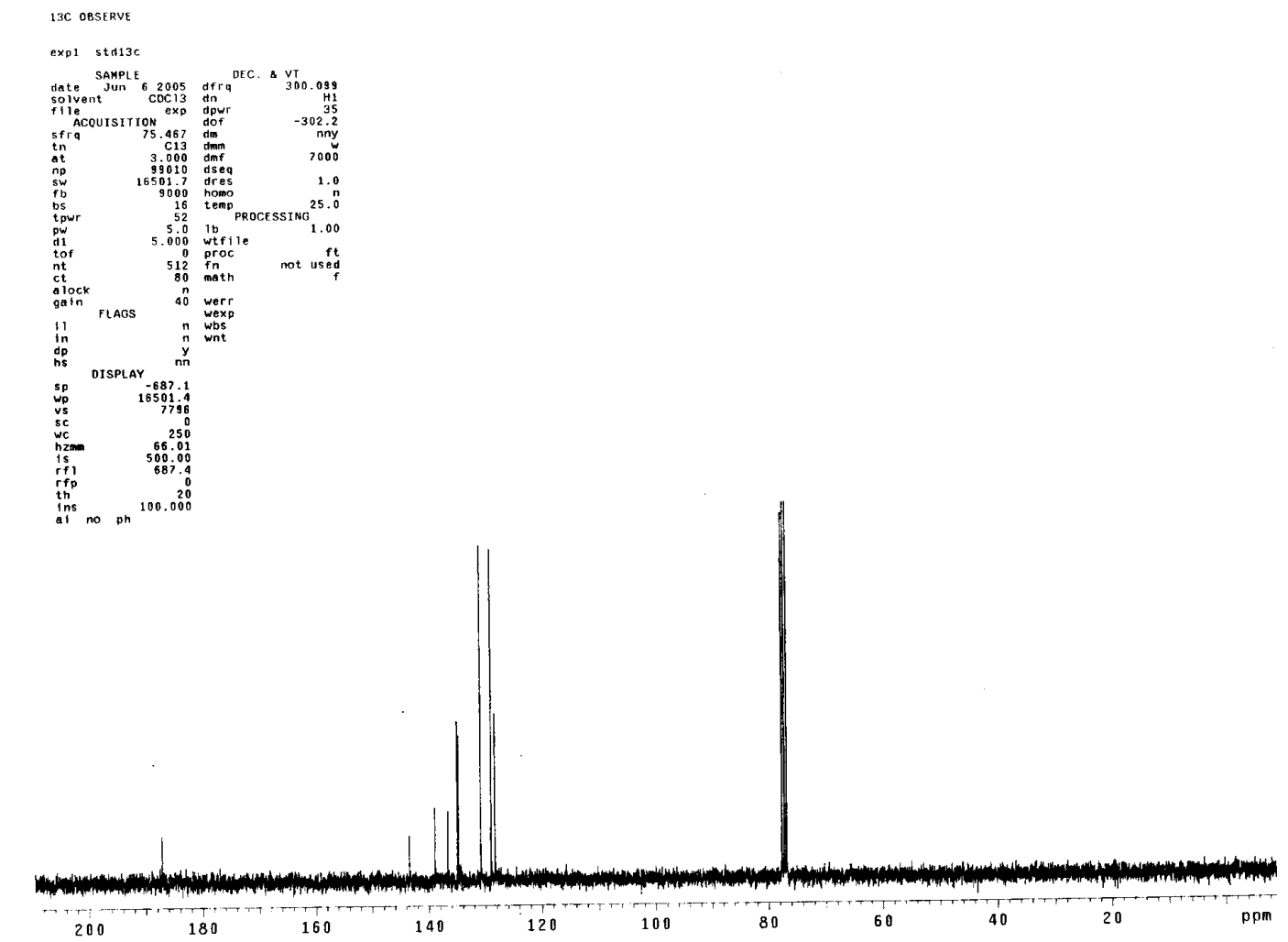

${ }^{1}$ H NMR and ${ }^{13}$ NMR spectra of 3-Chlorophenyl 2-thiophen ketone (33).
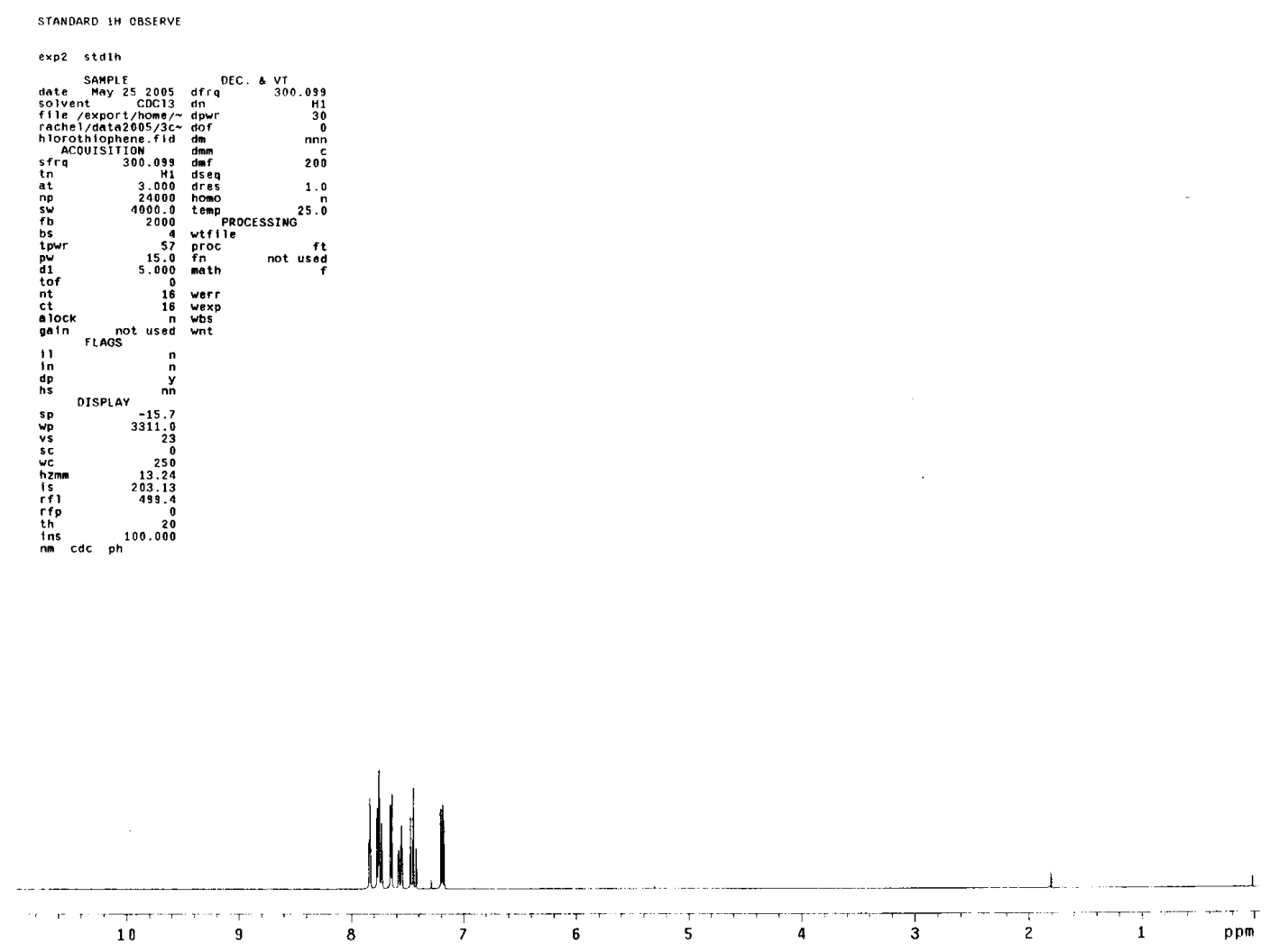


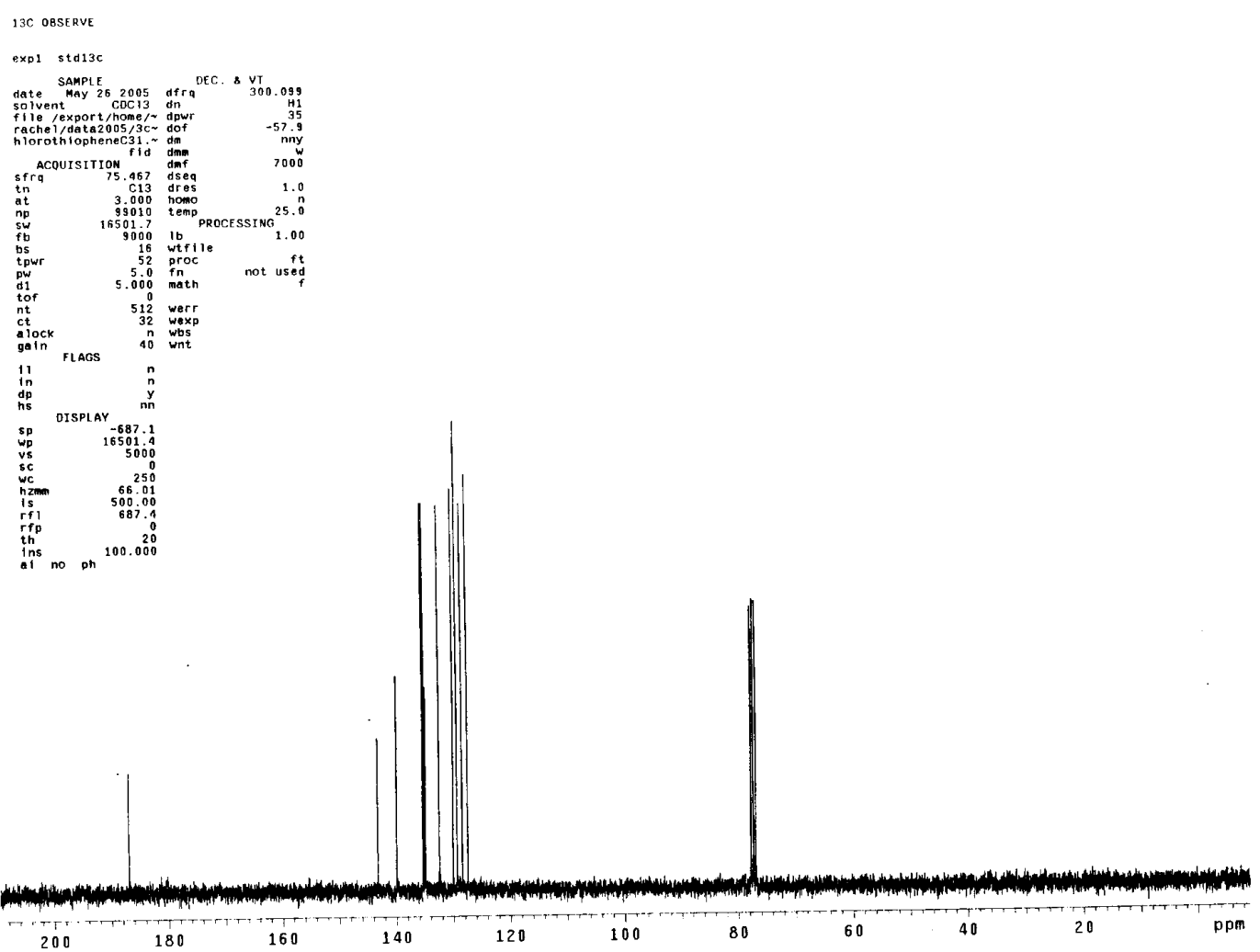

${ }^{1}$ H NMR and ${ }^{13}$ NMR spectra of 4-Bromophenyl 2-furan ketone (35).
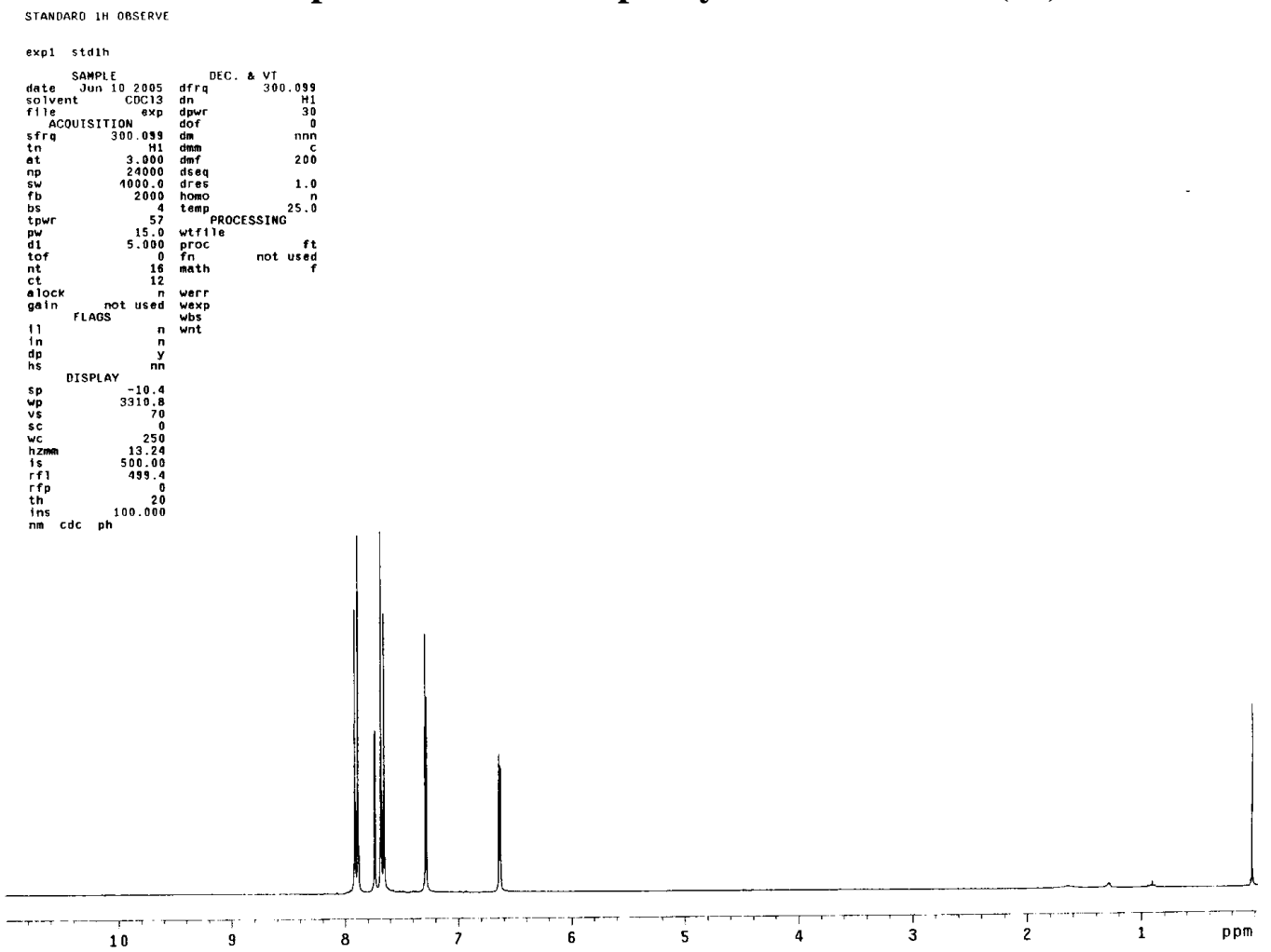


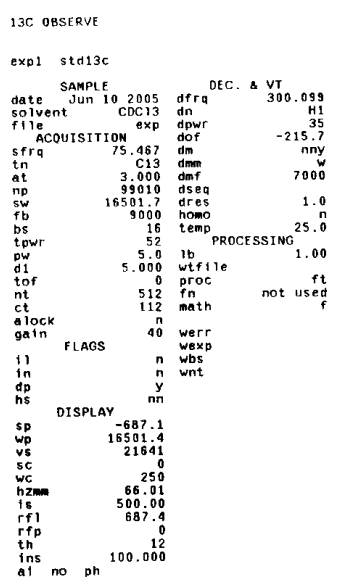



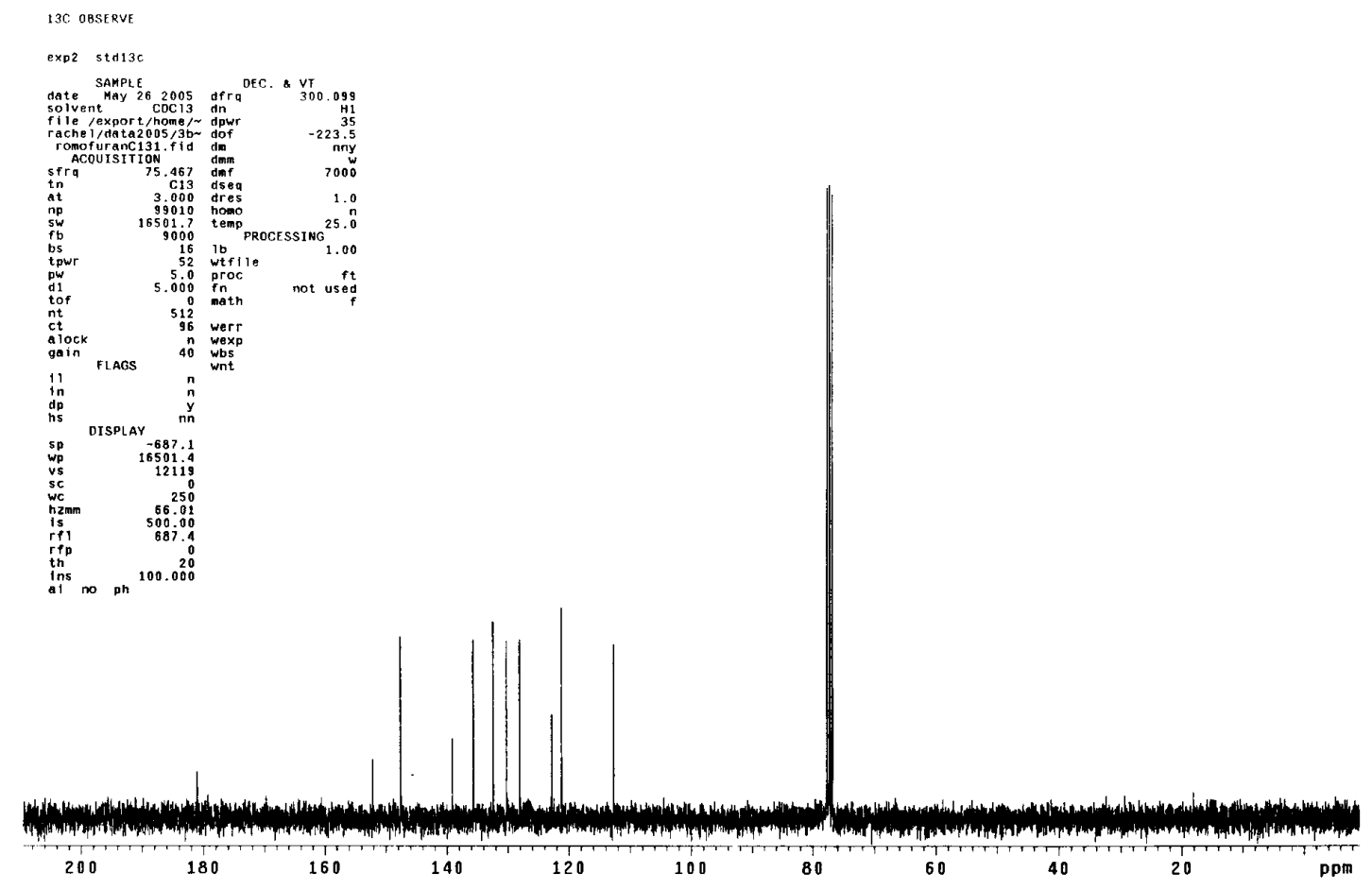

${ }^{1}$ H NMR and ${ }^{13}$ NMR spectra of 2-Bromophenyl 2-furan ketone (39).
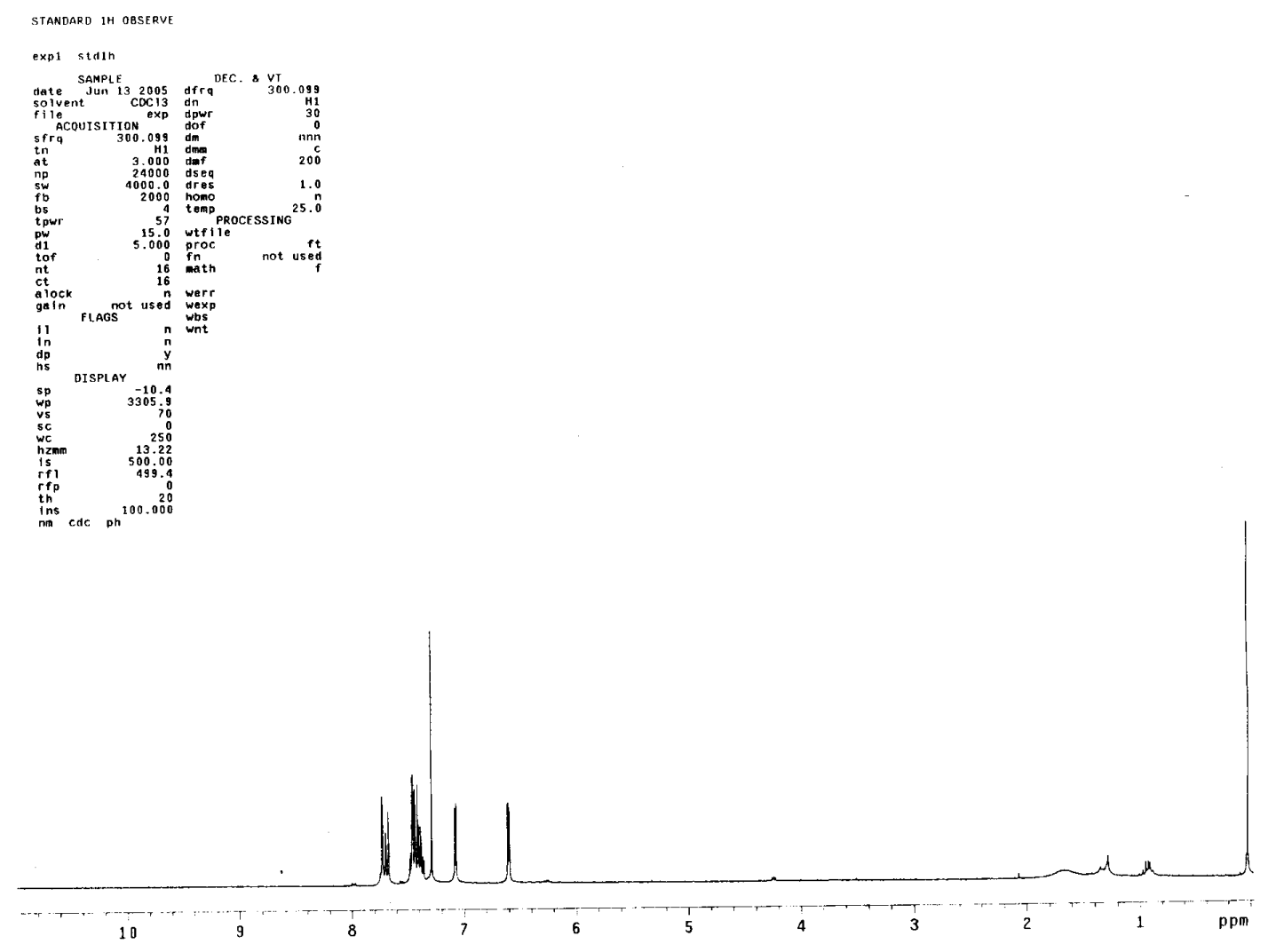


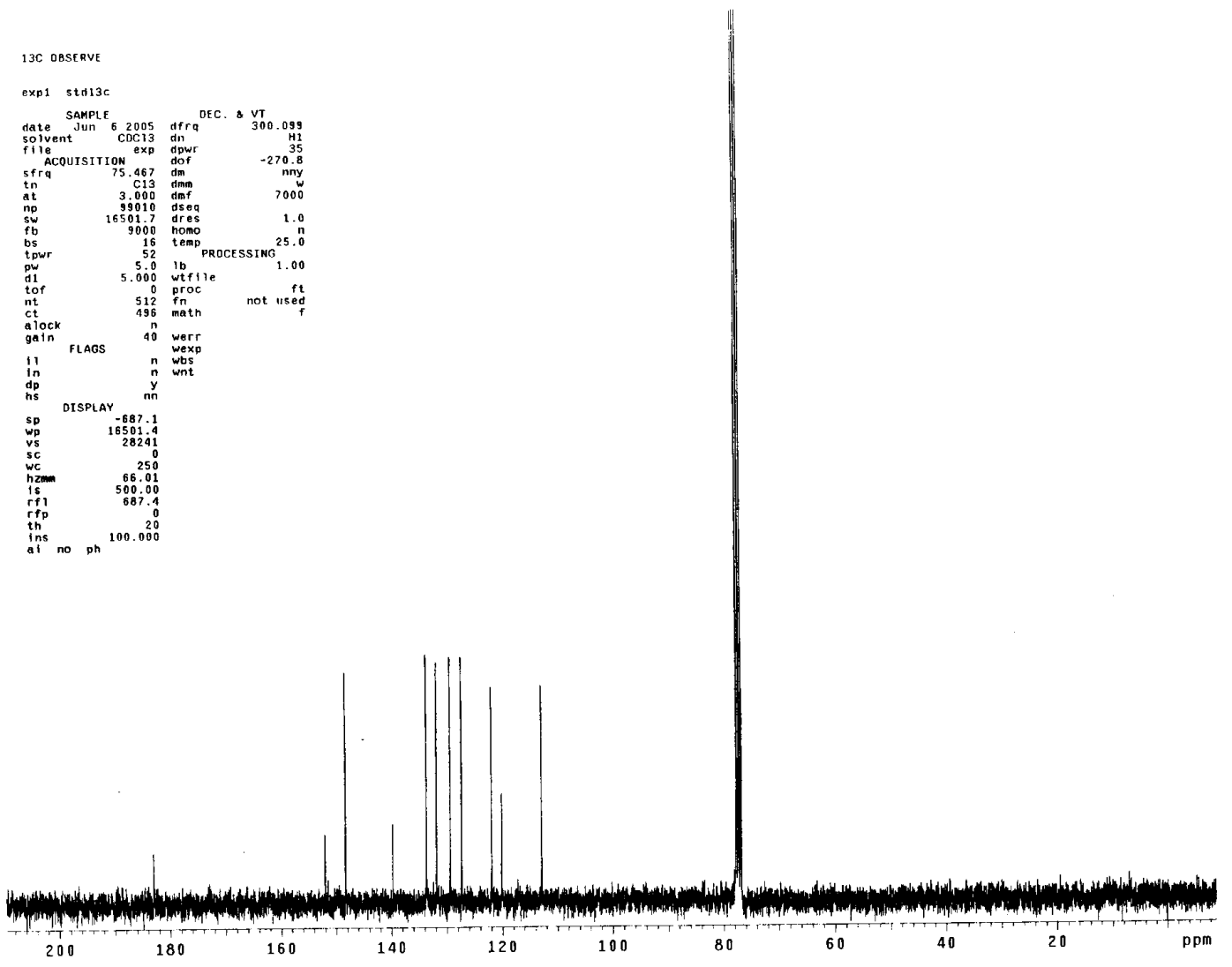

\section{References}

(1) Terao, Y.; Nomoto, M.; Satoh, T.; Miura, M.; Nomura, M. J. Org. Chem. 2004, 69, 69426944.

(2) Lee, P. H.; Lee, S. W.; Seomoon, D. Org. Lett. 2003, 5, 4963-4966.

(3) Kang, S. K.; Ryu, H. C.; Lee, S. W. J. Organomet. Chem. 2000, 610, 38-41.

(4) Tanake, M.; Kobayashi, T.; Sakakura, T.; Nippon Kagaku Kaishi 1985, 3, 537-546.

(5) Kang, S. K.; Ho, P. S.; Lee, J. C.; Lee, K. J. Synthesis 1998, 6, 823-825.

(6) Resser, M.; Maas, G. J. Org. Chem. 2004, 69, 4913-4924.

(7) Matsubara, H.; Yasuda, S.; Ryu, I. Synlett. 2003, 2, 247-249.

(8) Heathcock, C. H.; Gulick, L. G.; Dehlinger, T. J. Heterocycl. Chem. 1969, 6, 141-142.

(9) Jeon, K, O.; Jun, J. H.; Y, J. S.; Lee, C. K. J. Heterocycl. Chem. 2003, 40, 763-771. 
(10) Mohamed Ahmed, M. S.; Mori, A. Org. Lett. 2003, 5, 3057-3060.

(11) Pucheau, M.; Darses, S.; Genet, J. P. J. Am. Chem. Soc. 2004, 126, 15356-15357. 